\title{
The Xenopus Amygdala Mediates Socially Appropriate Vocal Communication Signals
}

\author{
Ian C. Hall, ${ }^{1}$ Irene H. Ballagh, ${ }^{2}$ and Darcy B. Kelley ${ }^{1,2}$ \\ ${ }^{1}$ Biological Sciences and ${ }^{2}$ Neurobiology and Behavior, Columbia University, New York, New York 10027
}

Social interaction requires that relevant sensory information is collected, classified, and distributed to the motor areas that initiate an appropriate behavioral response. Vocal exchanges, in particular, depend on linking auditory processing to an appropriate motor expression. Because of its role in integrating sensory information for the purpose of action selection, the amygdala has been implicated in social behavior in many mammalian species. Here, we show that two nuclei of the extended amygdala play essential roles in vocal communication in the African clawed frog, Xenopus laevis. Transport of fluorescent dextran amines identifies the X. laevis central amygdala (CeA) as a target for ascending auditory information from the central thalamic nucleus and as a major afferent to the vocal pattern generator of the hindbrain. In the isolated (ex vivo) brain, electrical stimulation of the CeA, or the neighboring bed nucleus of the stria terminalis (BNST), initiates bouts of fictive calling. In vivo, lesioning the CeA of males disrupts the production of appropriate vocal responses to females and to broadcasts of female calls. Lesioning the BNST in males produces an overall decrease in calling behavior. Together, these results suggest that the anuran CeA evaluates the valence of acoustic cues and initiates socially appropriate vocal responses to communication signals, whereas the BNST plays a role in the initiation of vocalizations.

\section{Introduction}

Acoustic communication requires the coordination of auditory processing and vocal motor production, but brain nuclei that integrate auditory information for the purpose of action selection remain understudied. Across species, the extended amygdala, a multinucleus complex including the central amygdala (CeA) and bed nucleus of the stria terminalis (BNST), modulates many social behaviors (Newman, 1999; Goodson and Kabelik, 2009; O'Connell and Hoffman, 2011). The most abundant examples of auditory-amygdalar control of behavior are fear conditioning paradigms using negatively valenced stimuli (Rodrigues et al., 2009; Johansen et al., 2011). However, neurons in the mammalian basolateral amygdala also receive auditory input and respond to communication calls (Naumann and Kanwal, 2011; Peterson and Wenstrup, 2012). Here, we describe a potential conserved role for the amygdala in vocal communication in the African clawed frog, Xenopus laevis.

Vocal interactions are the dominant mode of social communication in X. laevis. Behavioral responses to playbacks of communication calls mirror responses to calls from partners (Zornik and Kelley, 2011). Male X. laevis attract females and suppress the

\footnotetext{
Received March 10, 2013; revised Aug. 1, 2013; accepted Aug. 3, 2013.

Author contributions: I.C.H., I.H.B., and D.B.K. designed research; I.C.H. and I.H.B. performed research; I.C.H. and I.H.B. analyzed data; I.C.H., I.H.B., and D.B.K. wrote the paper.

This work was supported by National Institutes of Health F32 GM103266 to I.C.H., a Fullbright International Science \& Technology Graduate Fellowship to I.H.B., and National Institutes of Health R01 NS23684 to D.B.K. We thank Benjamin Matthews for advice on image processing and statistical analysis and Charlotte Barkan, Elizabeth Leininger, and Martha Tobias for helpful comments on the manuscript.

The authors declare no competing financial interests.

Correspondence should be addressed to Dr. Ian C. Hall, Columbia University, Biological Sciences, 913 Fairchild Center, MC 2430, New York, NY 10027. E-mail: ich2105@columbia.edu.

DOI:10.1523/JNEUROSCI.1190-13.2013

Copyright $\odot 2013$ the authors $\quad 0270-6474 / 13 / 3314534-15 \$ 15.00 / 0$
}

calling of nearby males with bouts of advertisement calling: a repeated, biphasic pattern of alternating fast and slow trills (Tobias et al., 1998) (Fig. 1). In the presence of another frog, sexually receptive males will also produce answer calls, a modified advertisement call, distinguished by higher intensity clicks and briefer slow trills, especially if the other frog is female and communicates her sexual receptivity with the female-specific rapping call (Tobias et al., 1998, 2004). Sexually unreceptive females produce ticking, a slower train of clicks that temporarily suppresses male advertisement (Elliott and Kelley, 2007).

The click patterns that comprise different calls are produced by a hindbrain vocal pattern generator (VPG), which includes the pretrigeminal nucleus of the dorsal tegmental area of the medulla (DTAM) and the vocal motor nucleus (VMN; equivalent to the mammalian nucleus ambiguus) (Brahic and Kelley, 2003; Rhodes et al., 2007). Clicks are created by individual contractions of laryngeal muscles driven by brief compound action potentials (CAPs) produced by vocal motor neurons. The temporal pattern of CAP activity in the laryngeal nerve matches the pattern of clicks in calls in vivo (Yamaguchi and Kelley, 2000), and ex vivo in the isolated brain. Fictive calling can be stimulated ex vivo by bath application of serotonergic agents or electrical stimulation of DTAM (Rhodes et al., 2007; Yu and Yamaguchi, 2009, 2010).

Auditory information is conveyed from the ear to hindbrain nuclei and the inferior colliculus (IC, or laminar nucleus of the torus semicircularis) (Kelley, 1980, 1981). From the midbrain, ascending auditory signals are conveyed to the telencephalon via a thalamic multisensory area, the central thalamic nucleus (CT) (Edwards and Kelley, 2001; Endepols and Walkowiak, 2001; Carr and Eds-Walton, 2008).

Here, we provide anatomical evidence for the connection of the auditory and vocal circuits through the anuran homolog to 

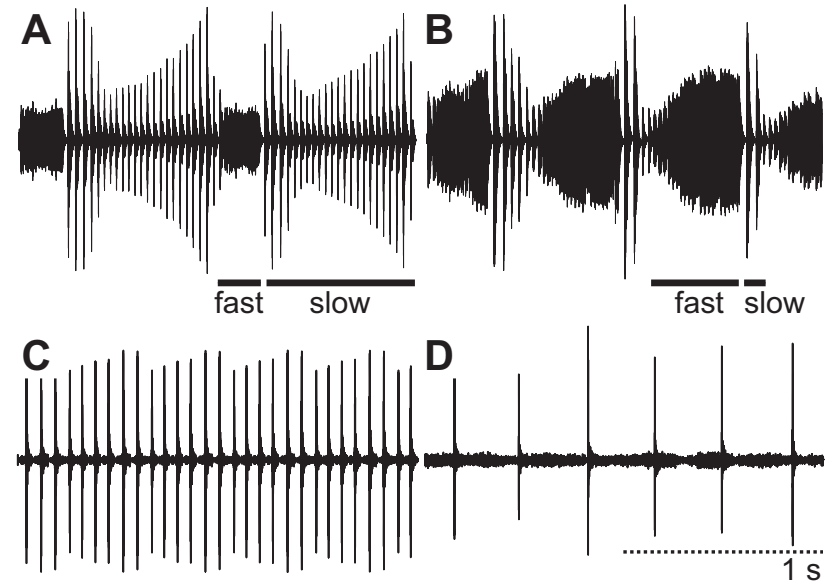

Figure 1. Example oscillograms of the male and female $X$. laevis calls described in this study. For male advertisement $(\boldsymbol{A})$ and answer calls $(\boldsymbol{B})$, the fast and slow trill phase of a single call are underlined. Female rapping $(\boldsymbol{C})$ and ticking $(\boldsymbol{D})$ communicate sexual receptivity or nonreceptivity, respectively. Each call segment is 2 s in duration.

the CeA. We demonstrate that electrical stimulation of the CeA and BNST ex vivo results in fictive vocal activity and that lesioning the CeA or BNST in vivo alters social vocal behavior.

\section{Materials and Methods}

\section{Animals}

Sexually mature male $X$. laevis were obtained from Xenopus Express, Nasco, or Xenopus I and group housed in polycarbonate aquaria under a 12/12 light-dark cycle. Frogs were fed brittle (Nasco) and had their water changed twice per week. The frogs used in these experiments weighed between $22.1 \mathrm{~g}$ and $76.6 \mathrm{~g}$ (mean, $43.2 \mathrm{~g}$ ) with snout-vent lengths between $5.7 \mathrm{~cm}$ and $9.1 \mathrm{~cm}$ (mean, $7.4 \mathrm{~cm}$ ). All animals were housed and handled in accordance with the guidelines established by the Columbia University Animal Care and Use Committee.

\section{Isolated brain preparation}

Anatomical and electrophysiological experiments were performed using an isolated (ex vivo) brain preparation (Rhodes et al., 2007; Zornik and Kelley, 2008; Zornik et al., 2010). Frogs were deeply anesthetized with an $0.95 \mathrm{ml}$ injection of $1.3 \%$ tricaine methyl sulfonate (MS-222; Sigma) into the dorsal lymph sac, placed on ice for $10 \mathrm{~min}$, and then transcardially perfused with $30-50 \mathrm{ml}$ of an oxygenated $\left(99 \% \mathrm{O}_{2} / 1 \% \mathrm{CO}_{2}\right)$ ice-cold saline solution ( $\mathrm{pH} 7.8$; in mM as follows: $96 \mathrm{NaCl}, 20 \mathrm{NaHCO}_{2}, 2 \mathrm{CaCl}_{2}$, $2 \mathrm{KCl}, 0.5 \mathrm{MgCl}_{2}, 10 \mathrm{HEPES}$, and 11 glucose; Sigma) followed by decapitation. The brain was promptly dissected from the skull in ice-cold saline and pinned in a silicone elastomer-lined (Sylgard; Dow Corning) 140 $\mathrm{mm}$ Petri dish, and the arachnoid layer of the meninges removed.

Anatomy. For dye-tracing experiments, the pia was removed from either the entire brain (for whole-mount brains), or simply over the site(s) injected with fluorescent dye (for sectioned brains). After tracer application, brains were transferred to $500 \mathrm{ml}$ of oxygenated saline and kept at $4^{\circ} \mathrm{C}$ in the dark for $3-5 \mathrm{~d}$. The oxygenated saline was replaced every $24 \mathrm{~h}$ (Zornik and Kelley, 2007).

Electrophysiology. For electrophysiological recording, the pia over either the dorsal or ventral surface of the telencephalon was removed to facilitate electrode penetration. To access sites in caudal subpallium, where both BNST and CeA are located, the electrode was inserted ventrally with the brain inverted, or dorsally, which required transecting the septum of both hemispheres from rostral to caudal, close to the boundary between the dorsal septum and the medial pallium. After dissection, brains equilibrated at room temperature for $1-3 \mathrm{~h}$ in constantly oxygenated saline. Before recording, brains were transferred to an $85 \mathrm{~mm}$ silicone elastomer-lined Petri dish, filled with $\sim 40 \mathrm{ml}$ of room temperature saline, and secured with minutien pins (Fine Science Tools) placed in the olfactory nerves and the spinal cord. In brains in which the septum had been transected, 2 more minutien pins were placed in the pallium to expose the ventricular surface of the subpallium. Room temperature oxygenated saline was superfused through the recording dish at a rate of $>150 \mathrm{ml} / \mathrm{h}$, except during periods of pharmacological stimulation (see below).

\section{Fluorescent dye injection}

Tracer application methods have been described in detail previously (Zornik and Kelley, 2007). Briefly, dextran-conjugated fluorescent dyes were applied either via the tip of dye-coated minutien pins or were dissolved in saline solution $(10-20 \% \mathrm{w} / \mathrm{vol})$ and delivered via pressure injection (4-10 pulses, $20-25$ psi, $20-30 \mathrm{~ms}$; Pico-spritzer II; General Valve) through a pulled glass pipette broken back to a tip diameter of $20-25 \mu \mathrm{m}$. We used three dextran-conjugated dyes in these experiments: tetramethylrhodamine-, fluorescein-, and AlexaFluor-488conjugated dextran, all anionic, lysine-fixable, and 10,000 molecular weight (Invitrogen).

Injection coordinates. CeA neurons are located in the caudal-most third of the ventral subpallium (Moreno and González, 2005). As with all striatal-derived nuclei in the anuran telencephalon, CeA cell bodies are densely packed and clustered just distal to the ventricular surface. The caudal boundary of CeA can be clearly distinguished in a lateral view, just anterior to the myelinated lateral forebrain bundle (LFB) as it crosses from telencephalon to diencephalon. Typically two or three injection sites, $400-500 \mu \mathrm{m}$ below the pial surface and spaced along the anteriorposterior extent of the nucleus $\sim 400 \mu \mathrm{m}$ apart, were most effective in repeating previously described connectivity patterns for this nucleus (Marín et al., 1998a; Moreno and González, 2005).

Injections of tracer into the thalamus used a dorsal approach and were placed just anterior to the pretectal commissure, midway between the midline and the lateral edge of the diencephalon, $500 \mu \mathrm{m}$ deep. The pattern of retrograde labeling of thalamic cells after striatal injections revealed a curved nucleus, with more posterior cells located ventrally within the diencephalon. Thus, in some cases, two injection sites, separated by $\sim 200 \mu \mathrm{m}$ with the more posterior site at a depth of $600 \mu \mathrm{m}$, were required.

Both dorsal and lateral approaches were used to inject the IC. For a dorsal approach, the 3-5 injection sites were spaced across the width of one hemisphere of the midbrain from the midline to the lateral edge of the torus, approximately halfway between the rostral and caudal margins of the midbrain, at a depth of $800 \mu \mathrm{m}$. For a lateral approach, we used a fine sulcus that delineates the boundary between the torus and the overlying tectum as a landmark, with 3-5 injection sites posterior to the sulcus, $600-800 \mu \mathrm{m}$ deep.

We used previously published coordinates for injections into DTAM (Rhodes et al., 2007; Zornik and Kelley, 2008). These injections were placed using a dorsal approach $600-900 \mu \mathrm{m}$ deep from the rostral edge of the cerebellum and just lateral to the edge of the fourth ventricle. VMN injections were most successful using dye recrystallized to $50-100 \mu \mathrm{m}$ diameter on the tips of minutien pins. Typically, $3-5$ sites, extending posteriorly in a $\sim 500 \mu \mathrm{m}$ column from the insertion point of the fourth nerve rootlet, sufficed to efficiently back-label DTAM neurons.

All injection sites were confirmed by comparison of the cytoarchitecture at the injection site to reference brains and previously published literature (Marín et al., 1998a; Edwards and Kelley, 2001; Brahic and Kelley, 2003; Moreno and González, 2005; Moreno et al., 2012). Although the size of the injection site and the volume of dye injected varied between brains, and larger injections were essential for obtaining sufficient fluorescence signal to resolve fine details of dendrites and axon arbors, all the connections we report between nuclei of auditory and vocal circuits and the CeA were also observed with smaller focal injections within the boundaries of the nuclei.

\section{Histology and immunohistochemistry for synaptophysin}

After 3-5 d in oxygenated saline, tracer-injected brains were transferred to $4 \%$ PFA in $0.01 \mathrm{~m}$ PBS (Sigma) and fixed for $24 \mathrm{~h}$. Brains that were processed as sections were cryo-protected in 30\% sucrose, sectioned in the horizontal or transverse plane at $22 \mu \mathrm{m}$ on a cryostat (Hacker Instrument), counterstained with 300 nM DAPI (Invitrogen), serially dehydrated in ethanol, cleared in xylenes, and coverslipped with Cytoseal XYL (Richard-Allan Scientific). 

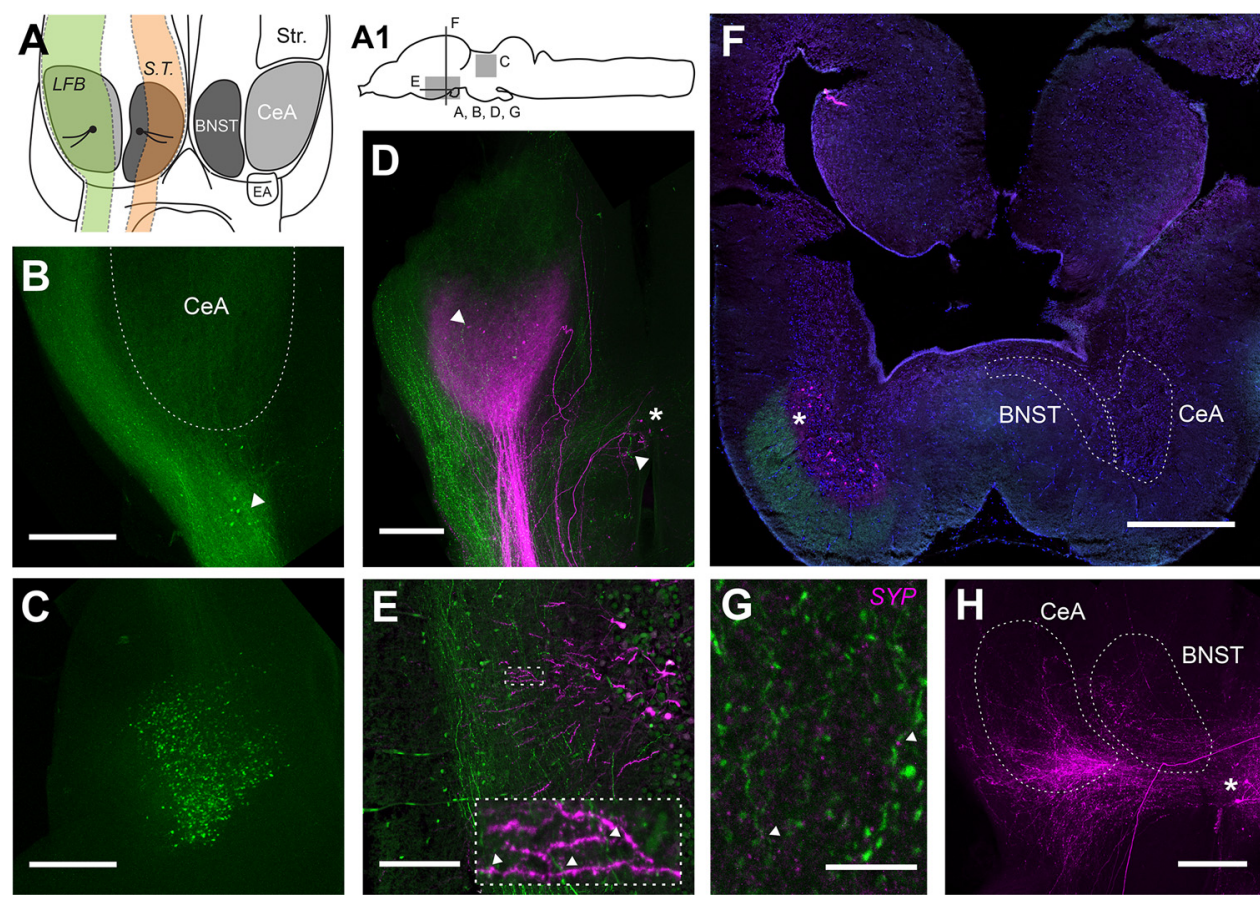

Figure 2. CeA and BNST in X. laevis. A, Schematic view of the caudal portion of the ventral telencephalon in X. laevis, viewed from below (rostral up). The boundaries of BNST and CeA are indicated on each hemisphere, with their respective associated fiber pathways overlaid on the left hemisphere: green represents LFB; orange, ST [stria terminalis]. Diagram single neurons in each nucleus show the typical orientation of cell bodies and dendrites in the nucleus with respect to the forebrain ventricle (not pictured) and the fiber tract. In the right hemisphere, the location of the adjacent nuclei of the basal ganglia is shown in white: Str., Striatum; EA, entopeduncular area, a pallidal homolog. $A_{1}$, Schematic view of the entire brain of $X$. laevis in the sagittal orientation, showing the location of sections (lines) and whole-mount views (gray boxes) from subsequent parts of the figure. $\boldsymbol{B}$, LFB and axon terminal field within the ventral subpallium labeled by CT injection in a whole-mount brain. $z$-stackimage ( $500 \mu \mathrm{m}$ thick) through caudal ventral telencephalon (horizontal plane, rostral up). The CeA is outlined by a white overlay. Arrowhead indicates back-labeled EA cells. Scale bar, $250 \mu \mathrm{m}$. C, CT cells back-labeled by CeA injection in a whole-mount brain. $z$-stack image (500 $\mu \mathrm{m}$ thick) through dorsal thalamus (horizontal plane, rostral up). Scale bar, $250 \mu \mathrm{m}$. D, Caudal telencephalon in a whole-mount brain after double-label with CT (green) and DTAM (magenta) injections. $z$-stack image ( $375 \mu \mathrm{m}$ thick) in the horizontal plane (rostral up). Arrowheads indicate back-labeled DTAM-projecting cells (magenta). Although the green (T axons extend into the more rostrally located striatum, the DTAM terminal field and DTAM-projecting cells are restricted to the caudal portion of the subpallium. No back-labeled cells are seen in EA after DTAM injection. Axons connecting CeA and DTAM are also contained within the LFB, but in a more medial location than CT axons (green). Back-labeled cells (both magenta and green) can also be seen in APOA (asterisk). Scale bar, $250 \mu \mathrm{m}$. E, High-power (40X) image of anterogradely labeled (TT axons (green) within the LFB interacting with locally labeled CeA dendrites (magenta). Horizontal section, rostral up. Scale bar, $50 \mu \mathrm{m}$. Dashed boxes indicate the areas shown at expanded magnification in the inset. Arrowheads indicate three example sites of putative synaptic contacts between boutons and spines. $\boldsymbol{F}$, Transverse section through caudal telencephalon just rostral to APOA after double-label with CT (green) and DTAM (magenta) injections. The CT axon terminal field is clearly restricted to the outer half of the CeA dendritic fields and neatly overlaps the more proximally located terminal field (asterisk) and cell bodies back-labeled by DTAM injection. The combination of DTAM and CT labeling delineates the border of CeA with the BNST medially and the medial amygdala dorsally (see white overlay on contralateral side). Scale bar, $500 \mu \mathrm{m}$. G, Synaptophysin (SYP) puncta (magenta) located on CT axons (green). High-power (63X) $z$-stack image (4.5 $\mu \mathrm{m}$ thick) in the horizontal plane (rostral up). Arrowheads indicate two example puncta located on axons labeled by tracer injection into CT. Scale bar, $10 \mu \mathrm{m}$. $\boldsymbol{H}$, Axon terminal fields within CeA and BNST labeled by contralateral CeA injection. $z$-stack image (400 $\mu \mathrm{m}$ thick) in the horizontal plane (rostral up). White dashed overlay outlines the BNST area as distinct from CeA. The projection from CeA is bilateral; however, terminal fields within the ipsilateral hemisphere are obscured by the proximity of the dye injection. Back-labeled cells within APOA can be seen at lower right (asterisk). Scale bar, $250 \mu \mathrm{m}$.

In a subset of three brains with injections into CT, or into CeA and VMN, fluorescence-conjugated immunohistochemistry for synaptophysin was used to identify presynaptic specializations. Briefly, fixed frozen sections $(22 \mu \mathrm{m})$ were thaw-mounted onto Superfrost Plus slides (VWR), rinsed in $0.01 \mathrm{M}$ PBS, and blocked for $1 \mathrm{~h}$ in $0.3 \%$ Triton-X/PBS (PBT; Triton X-100 from Fisher Scientific) and 1\% normal donkey serum (Jackson ImmunoResearch Laboratories). Mouse monoclonal anti-synaptophysin [SY38] antibody (Abcam) was prepared at a concentration of 1:150 in blocking solution. Tissue was incubated in the primary antibody solution at $4^{\circ} \mathrm{C}$ for two nights. Slides containing alternate sections were incubated in blocking solution without primary antibody for the same period to test for antibody specificity. Sections were washed in PBT and then incubated with a donkey anti-mouse secondary antibody conjugated to AlexaFluor-647 (1:400; Jackson ImmunoResearch Laboratories) at room temperature for $2.5 \mathrm{~h}$, washed again in PBT $(2 \times 5 \mathrm{~m})$ before counterstaining with DAPI, and dehydration as described above.

Whole-mount brains were serially dehydrated in ethanol and cleared with methyl salicylate. Because this procedure causes approximately threefold shrinkage in brains, scale bars for whole-mount images in Figure 2 do not reflect distances in live tissue. Whole-mount brains were viewed in a depression slide immersed in methyl salicylate.

\section{Fluorescent imaging}

Brains were visualized on either a Zeiss 510 or a Zeiss 710 confocal microscope using $40 \times$ Plan Neofluar 1.3 N.A., EC $10 \times$ Plan Neofluar $0.30 \mathrm{NA}$, and Plan-Apochromat $63 \times 1.40$ Oil DIC M27 lenses (Carl Zeiss). Twelve-bit $z$-stack images were collected using Zeiss Zen LE software at $0.57 \mu \mathrm{m}(63 \times$ objective, sectioned tissue $), 1 \mu \mathrm{m}$ ( $40 \times$ objective, sectioned tissue), $11 \mu \mathrm{m}$ (10× objective, sectioned tissue), or 15-25 $\mu \mathrm{m}$ $(10 \times$ objective, whole-mount) increments. When ROIs could not be scanned in a single frame, multiple images of adjacent areas were assembled using Photoshop CS2 (Adobe Systems) or by using the tiling function on the Zeiss 710 microscope. $z$-stack images were compiled and converted to $z$-projections using Fiji/ImageJ (National Institutes of Health). Where necessary, the Despeckle tool was used to remove image noise, and the brightness and contrast of the image were optimized for presentation using the Color Balance function. These tools were not used when images were used for quantification.

Synaptophysin image analysis. In a $22 \mu \mathrm{m}$ horizontal section that had been incubated with synaptophysin primary antibody, we collected a $z$-stack image of 10 sections at $0.57 \mu \mathrm{m}$ spacing through rostral CeA at $63 \times$. A parallel procedure that omitted the primary antibody was applied to the corresponding area within the CeA of the immediately preceding 
section, using the same settings for laser power and gain to take the image. $z$-stack images were flattened using the maximum intensity $z$-project function in Fiji (National Institutes of Health).

To measure puncta density per unit area, ROIs were selected from within the LFB zone of CeA in sections stained for synaptophysin, with an equivalent area selected from the same region from the immediately preceding nonprimary incubated section. The antibody channel from both images was converted to an 8-bit grayscale image, thresholded at the same value, and then measured using the analyze particles function of Fiji. Particles with total area $<0.03 \mu \mathrm{m}^{2}$ represent noise and were excluded, and the number of remaining particles was divided by the total area of the ROI $\left(\mu \mathrm{m}^{2}\right)$ and then multiplied by 100 to obtain a value of density of puncta per $100 \mu \mathrm{m}^{2}$.

To measure puncta per unit axon length, axons within rostral CeA labeled with AlexaFluor-488 (Jackson ImmunoResearch Laboratories) after dye injection into CT were traced by a "blind" observer using the segmented line Region of Interest tool in Fiji within the dye channel. Equal lengths of axons within CeA were traced from both primaryantibody-incubated sections and the adjacent nonprimary-incubated section. To include the entire width of the axon, the line selection was expanded to include $0.5 \mu \mathrm{m}$ surrounding the traced line, and puncta were counted for the expanded line selections within the antibody channel for the same image, as described above for area analysis. The number of puncta $>0.03 \mu \mathrm{m}^{2}$ within the selected area for each image was divided by the total length of all traced axons for each section and then multiplied by 100 to obtain a value of puncta per $100 \mu \mathrm{m}$ length of axon.

\section{Ex vivo electrophysiology}

As previously described (Rhodes et al., 2007; Zornik and Kelley, 2008; Zornik et al., 2010), fictive vocalizations were recorded from the posterior-most rootlet of cranial nerve IX-X (N.IX-X) of isolated brains using a suction electrode. Nerve activity was recorded throughout the entire duration of the experiment (typically $7 \mathrm{~h}$ ). A tungsten microelectrode ( $15 \mathrm{M} \Omega$ resistance, FHC) was inserted into the telencephalon (one site per brain) and connected to a stimulator (model S88; Grass Instruments) and constant current stimulation isolation unit (PSIU6; Grass Instruments). Nerve activity was amplified $1000 \times$ using a differential amplifier (model 1800; A-M Systems), digitized at $10 \mathrm{kHz}$ (Digi-data 1322A; Molecular Devices), and recorded on a PC using Clampex software (Molecular Devices).

Electrical and pharmacological stimulation of the telencephalon. Stimulus pulses (either short pulse: $0.1-0.5 \mathrm{~ms}$, or long pulse: $1-2 \mathrm{~ms}$ duration, $150 \mu \mathrm{A}$ ) were applied in trains. To explore the response characteristics of each preparation, trains varied in pulse repetition rate $(10-100 \mathrm{~Hz})$ and duration (1-10 s). For sites outside the CeA, stimulus rates and durations were deliberately chosen to match those most effective within the CeA or increased in duration to ensure maximum probability of eliciting a response. Over the course of $20 \mathrm{~min}, 1-10 \mathrm{~s}$ stimulus trains were repeated every $2 \mathrm{~min}$. In rare cases, stimulation episodes were terminated prematurely if N.IX-X was highly active or went into a prolonged period without spontaneous activity, and stimulus trains that occurred during these prolonged periods of inactivity were not included in the analyses.

As a positive control, bath application of serotonin hydrochloride (5HT; Sigma-Aldrich) was used to elicit fictive advertisement calling (Rhodes et al., 2007). 5-HT was applied directly to the recording chamber to a final concentration of $30 \mu \mathrm{M}$. After $\sim 3 \mathrm{~min}$, superfusion of fresh oxygenated saline was reinstated. 5-HT application was repeated at intervals of $1 \mathrm{~h}$ or interleaved with stimulation episodes.

After four stimulation episodes and at least two applications of 5-HT, interleaved pseudo-randomly with at least $50 \mathrm{~min}$ intervals between each application or stimulation episode, the stimulation site was marked for histological analysis by electrolytic lesion (single pulse stimuli: 250-1000 $\mathrm{ms}, 1.5 \mathrm{~mA}$ ). Each brain was allowed $20 \mathrm{~min}$ to recover after lesion, and then a final dose of 5-HT was applied. As published previously, focal lesions in the telencephalon did not interfere with the fictive calling response to 5-HT (Rhodes et al., 2007).

\section{Electrophysiological analysis}

Two types of laryngeal nerve activity were most frequently recorded after unilateral electrical stimulation of the telencephalon: desynchronized bursting and fictive calling. The criterion for stimulation-induced bursting activity was an asynchronous burst or rapid increases in CAP rates $>100 \mathrm{~Hz}$ that occurred within the stimulus train. Without direct recording from motor neurons, episodes of desynchronized laryngeal motor neuron activity cannot be easily distinguished from fictive breathing activity occurring in glottal motor neurons whose axons are also contained within the posterior nerve rootlet of N.IX-X. We thus measured all desynchronized activity episodes occurring within the period of stimulation in our analysis without attempting to distinguish desynchronized calling from fictive breathing or other forms of nerve activity. Using Clampfit software (Molecular Devices), we measured the occurrence, latency, and duration of desynchronized bursting activity coincident with the stimulus train. The beginning and end of a desynchronized episode were identified as the points at which the CAP rate exceeded and decreased $<100 \mathrm{~Hz}$, respectively.

Fictive advertisement calls are readily distinguished from other laryngeal nerve activity patterns (e.g., fictive breathing) by the high degree of synchronicity of their waveforms (Figure $3 B-D$, insets). The temporal pattern of CAPs mirrors the acoustic structure of the communication call produced in vivo (Yamaguchi and Kelley, 2000). Male advertisement and answer calls are composed of segments with two distinct interclick intervals: the fast and slow trill (Tobias et al., 1998, 2004) (Fig. 1). The CAP activity identified as fictive advertisement calling is also composed of fast and/or slow segments, with inter-CAP intervals (ICIs) comparable to the interclick intervals of in vivo calls (Rhodes et al., 2007; Yamaguchi and Kelley, 2000; Yu and Yamaguchi, 2010; Zornik et al., 2010). Fictive fast trills, the hallmark of 5-HT-induced fictive calling, are readily identified by the more than twofold increase in CAP amplitude that characteristically accompanies the transition to shorter ICIs (Fig. $3 B, C$ ). ICIs were measured with Clampfit software (Molecular Devices) using the Threshold Search function. Because of variation in the quality of the suction recording, the threshold was optimized for each brain individually.

Brains were only considered for further analysis if they responded to 5-HT application with fictive advertisement calling at least twice during the course of the experiment or if they produced at least one episode of fictive calling after telencephalon stimulation. Twenty-eight brains met one or both of these criteria, whereas seven brains failed to meet either and were thus excluded from further analysis. To meet the criterion for fictive calling in response to 5-HT, a brain had to produce at least two high amplitude fictive fast trills during the $5 \mathrm{~min}$ period of 5-HT application and washout. Latency to 5-HT-induced calling was measured from the electrical artifact marking the start of 5-HT introduction to the bath to the beginning of the first fictive fast trill. For each 5-HT application, no more than 10 advertisement calling cycles (10 fictive fast trills, and the intervening introductory/slow phases between them) were measured for ICI analysis. In cases where $>10$ fictive fast trill segments were produced, a block of 10 consecutive advertisement calls was chosen from the middle of the calling bout for analysis.

The criterion for a stimulation-induced fictive calling episode was a fictive fast or slow trill of $>15$ consecutive CAPs with ICIs $<50 \mathrm{~ms}$. All stimulation-induced calling CAPs within designated trills were measured for ICI analysis. Latency to stimulation-induced calling episodes was measured from the beginning of the stimulus train to the first distinct calling CAP, and the duration from this first CAP to the last distinct calling CAP was measured. CAP activity produced by stimulation was scored as fast trill (monophasic, mean sustained ICIs $<20 \mathrm{~ms}$ ), slow trill (monophasic, mean sustained ICIs $>20 \mathrm{~ms}$ ), or biphasic (alternating trills at both fast and slow rates, without intervening pauses or periods of desynchronized nerve activity). For each brain, the number of trains that elicited fictive calling was expressed as a proportion of the total number of stimulus trains delivered.

Frequency histograms of ICIs were constructed from the measured ICIs pooled across brains for each condition (5-HT, short, and long pulse stimulation) and fitted with two Gaussian curves using the normalmixEM function of the mixtools package written in $\mathrm{R}$ ( $\mathrm{R}$ Foundation) (Benaglia et al., 2009) to obtain measures for means and $\operatorname{SDs}(\mu 1, \mu 2, \sigma 1$, $\sigma 2)$ for fast and slow trill phases for each condition (5-HT: 26 brains, 53 5-HT applications; short pulse stimulation: 8 brains, 22 successful stimulus trains; long pulse stimulation: 5 brains, 21 successful stimulus 

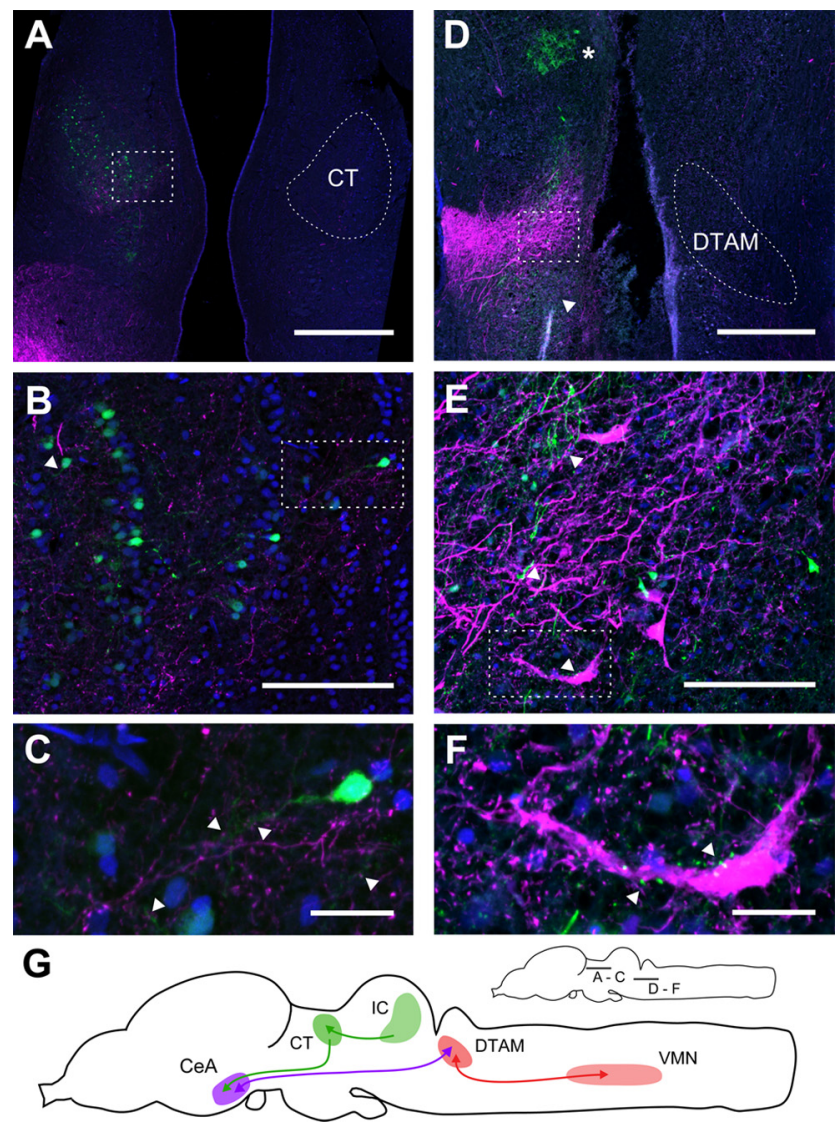

Figure 3. CeA connects to auditory and vocal circuits. $\boldsymbol{A}$, Horizontal section through the dorsal thalamus after CeA (green) and IC (magenta) injections (rostral up). Green back-labeled CeA-projecting cells overlap anterogradely labeled terminal field of IC axons (magenta) within CT (see white overlay on contralateral hemisphere). Magenta at lower left is a fiber tract that originates from the injection site (located just below the bottom of the panel). Scale bar, 500 $\mu \mathrm{m}$. $\boldsymbol{B}$, Expanded view of region indicated by the dashed box in $\boldsymbol{A}$, showing the overlap of back-labeled cells and the IC terminal field in greater detail. Proximal dendrites can be seen extending from back-labeled cell bodies (arrowhead). Blue channel is nuclear label from DAPI staining. Scale bar, $50 \mu \mathrm{m}$. $\boldsymbol{C}$, Expanded view of region indicated by the dashed box in $\boldsymbol{B}$, showing magenta axon boutons making apparent contact with proximal dendrites of green back-labeled CT cells (see arrowheads). Scale bar, $20 \mu \mathrm{m}$. D, Horizontal section through the tegmentum and DTAM after CeA (green) and VMN (magenta) injections (rostral up). VMN injection labels both cells and terminal fields within DTAM (dashed box) and overlaps with the green terminal field of labeled CeA axons. White overlay on the contralateral hemisphere outlines the boundaries of DTAM at this level. Green terminal fields from CeA are also visible in the more rostral pedunculopontine tegmental nucleus (asterisk) and the nucleus of the tractus solitarus (arrowhead) located just caudal to DTAM. Scale bar, $500 \mu \mathrm{m}$. E, Expanded view of region indicated by the dashed box in $\boldsymbol{D}$, showing overlap of back-labeled VMN-projecting cells and the CeA terminal field in DTAM. Instances of putative overlap of CeA axonal boutons (green) and VMN-projecting DTAM cells (magenta) are indicated by arrowheads. Blue channel is nuclear label from DAPI staining. Scale bar, $50 \mu \mathrm{m}$. $\boldsymbol{F}$, Expanded view of region indicated by $\boldsymbol{E}$, showing the overlap of axon boutons and proximal dendrites in greater detail. Scale bar, $20 \mu \mathrm{m}$. $\mathbf{G}$, $S$ chematic views of the entire brain of $X$. laevis in the sagittal orientation, showing the location of sections (lines) and a summary diagram of the connections described in Figures 1 and 2. Green represents auditory nuclei; red represents vocal nuclei.

trains). These results were compared with the results of a similar analysis performed on interclick intervals during episodes of in vivo advertisement calling (mean, $=51 \mathrm{~s})$ from frogs $(n=7)$ in the behavioral experiments described below.

To compare individual CAP properties between conditions, for each brain that produced at least one bout of fictive calling after forebrain stimulation, a subset of the CAPs elicited by 5 -HT and electrical stimulation was analyzed using Clampfit software ( 25 fast trill CAPs and 25 trill slow CAPs per condition for each brain). The peak amplitude $(\mathrm{mV})$, duration, time to peak, and half-width (all in ms) were measured manually for each CAP in Clampfit software. CAP duration was measured from the point at which the CAP deflection was distinguishable from baseline noise to the lowest point of the last trough before the CAP returned to baseline. Peak height and time to peak were determined from the first deflection point to the highest voltage value within the CAP duration. CAP half-width was the width of the CAP at half of the peak height. Because of consistent differences in the shape of CAPs from fast and slow trills across brains and because five brains produced only fast or slow CAPs in one or both of the two conditions (5-HT and electrical stimulation), fast and slow CAPs were considered separately in our statistical analysis.

Spontaneous episodes of fictive advertisement calling in the absence of 5-HT application or forebrain stimulation are extremely rare (3 of 28 brains in this study, each producing only one or two fictive advertisement calls each in the course of a $7 \mathrm{~h}$ experiment), and none of the 11 brains that produced fictive advertisement calls in the 2 min periods after stimulation was ever observed to produce spontaneous fictive advertisement calls in the intervals between stimulation episodes and 5-HT applications. However, spontaneous fictive amplectant calls, composed of two or three CAPs (Zornik and Kelley, 2008) and resembling vocalizations made by males when clasping another frog (Tobias et al., 2004), were commonly observed in the intervals between stimulation episodes and often persisted at a high, but variable, rate throughout the course of the experiment. As such, occasional instances of fictive amplectant calling observed during or after stimulus trains could not be distinguished from spontaneous nerve activity in the absence of stimulation, and so we did not consider them to represent stimulation-induced fictive calling.

Statistical analysis. Because call onset latencies were non-normally distributed, we used the Kruskal-Wallis one-way ANOVA to compare latencies between 5-HT, and short and long pulse stimulation. Differences between short and long pulse stimulation in the percentage of stimulus trains that elicited fictive calling and in the percentage of fictive calling responses that were biphasic were tested using the two-sample test of proportions. Analyses were performed using STATA (StataCorp), Prism (GraphPad), and R (R Foundation) software. Differences between measured peak height, width, half-width, and time to peak for slow and fast trill CAPs elicited by 5-HT and electrical stimulation were evaluated using paired $t$ tests on the mean values for each individual brain.

\section{In vivo behavioral recording}

Behavioral responses to acoustic playbacks and conspecific interactions were examined before and after lesions within the telencephalon. On the day before and the morning of behavioral testing, males were injected with human chorionic gonadotropin (100 IU/0.1 ml; Sigma-Aldrich) into the dorsal lymph sac to promote vocal behavior (Wetzel et al., 1983). Frogs were required to vocalize spontaneously when alone, for $15 \mathrm{~min}$ before behavioral testing.

Visual observations and sound recordings were performed in a $76.86 \mathrm{~m}^{3}$ fiberglass tank resting on a layer of foam, filled two-thirds with filtered water $\left(17-20^{\circ} \mathrm{C}\right)$, under dim red light. Vocalizations were recorded with a hydrophone (CA30, Aquaria Audio Products) and preamp (Omega SV, Lexicon Pro) onto a PC (Dimension 3000, Dell) running Goldwave software (Goldwave) in .WAV format. Frogs were retested behaviorally 6 or more days after surgery. If frogs failed to vocalize over 2 successive days of testing, they were tested again the following week for up to 3 weeks.

Playback stimuli. Conspecific vocalizations, male advertisement calls, female rapping calls, and female ticking calls (Fig. 1) were amplified (Realistic MPA30, Radio Shack) and presented through an underwater loudspeaker (UW-30 Diatran underwater loudspeaker, frequency 0.1-10 $\mathrm{kHz}$, University Sound). Except for rapping, all stimuli were obtained from recordings made by $\mathrm{ICH}$ in the recording tank described above; $X$. laevis female rapping (Fig. 1D) was obtained from field recordings in artificial ponds in Cape Town, Republic of South Africa (Tobias et al., 1998). At least two stimuli of each call type were compiled from three or more recordings of different frogs at comparable intensity, mixed, and trimmed to the desired playback duration. All stimuli were edited using Goldwave software (Goldwave). The choice of stimulus used for any 
individual frog was pseudorandom; the same stimulus was not used for the and postsurgical behavioral testing.

Each stimulus playback was flanked by a silent period of equal duration, providing prestimulus, during stimulus, and poststimulus calling periods of equal length. Based on previous behavioral studies (Tobias et al., 1998, 2004; Elliott and Kelley, 2007), the duration of the male advertisement playback was $15 \mathrm{~min}$; the duration of female rapping or ticking was $5 \mathrm{~min}$. The amplitude of stimuli was determined audio-visually; stimulus intensity was set at $75-100 \%$ of the intensity of the advertisement call of the focal male.

Conspecific interaction. An acoustic playback typically elicits only a portion of the full behavioral response to an actual conspecific (Tobias et al., 1998; Elliott and Kelley, 2007). Thus, as some males did not call postlesion in response to playbacks, we also recorded responses to conspecifics to determine whether the frogs were capable of vocal and other social behaviors. Because testing a male in a recording tank that previously contained a female increased his calling rates, conspecific interactions were recorded at the end of the recording day. After exposure to playback stimuli, a male or female frog was introduced to the tank containing the focal male and the pair was allowed to freely interact for 10 $\mathrm{min}$. The frog was removed and, after $5 \mathrm{~min}$, another frog of the opposite sex was introduced. Neither the male nor female stimulus frog was injected with human chorionic gonadotropin. The water in the tank was changed before the next set of playback tests.

\section{Calling analysis}

Audio recordings from playbacks and conspecific interactions were analyzed with Goldwave and Signal software (Engineering Design). The total time spent calling and the types of calls produced during each stimulus were scored. During playbacks, the time to call suppression (the cessation of calling for $>30 \mathrm{~s}$ ) and the time to the resumption of calling (duration of silence after initial suppression) were scored, as described previously (Elliott and Kelley, 2007).

\section{In vivo lesions in the extended amygdala}

Frogs were deeply anesthetized by submersion in 1\% MS-222 (Sigma), placed on ice for $10 \mathrm{~min}$, and the brain was accessed using a ventral approach. The CeA and BNST were distinguished using landmarks on the surface of the brain. Two types of lesions were made: excitotoxic and electrolytic. For excitotoxic lesions $(n=9)$, the CeA on each side of the brain received $0.3 \mu \mathrm{l}$ injections of ibotenic acid (IBO; Sigma; $0.1 \mu \mathrm{g} / 0.1$ $\mu \mathrm{l}$ frog saline) (Chambers and Lipska, 2011) over the course of $10 \mathrm{~min}$ via a pulled glass pipette, sealed to a Hamilton syringe. After injection, the syringe was left in place for $5 \mathrm{~min}$. Although IBO lesions spare fibers of passage (Schwarcz et al., 1979; Kohler and Schwarcz, 1983), the proximity of the CeA and BNST to the forebrain ventricles limited the volume and concentration of IBO that could be safely injected to produce reliable, isolated lesions, so electrolytic lesions were also produced. Electrolytic lesions ( $n=12$, CeA; $n=9$, BNST) were placed bilaterally by delivering $100 \mu \mathrm{A}$ of current via a monopolar tungsten electrode and a reference electrode in contact with the skin. One to three locations within a nucleus were targeted. The duration of current application varied from 15 to $60 \mathrm{~s}$ per site. Sham lesions were produced with bilateral saline injection into the CeA using the same procedure as the IBO injections $(n=6)$, or with bilateral $60 \mathrm{~s}$ current injection anterior to the CeA and BNST ( $n=3$, one each in the striatum, medial forebrain bundle, or LFB) using the same procedure as the electrolytic lesions. After lesioning, the surgical site was covered with Gelfoam (Pfizer) and sealed with Vetbond $(3 \mathrm{M})$. Frogs recovered for at least $6 \mathrm{~d}$ after surgery before behavioral testing.

\section{Histology}

After behavioral testing, animals were transcardially perfused with $4 \%$ PFA and the brain was removed and cryo-protected in 30\% sucrose. Brains used for ex vivo electrophysiology experiments were immersed in $4 \%$ PFA for $24-48 \mathrm{~h}$ before cryo-protection in 30\% sucrose. All brains were processed in $30 \mu \mathrm{m}$ transverse sections in a cryostat, Nissl-stained with cresyl violet, dehydrated through graded ethanol, and cleared with xylene before coverslipping. Sections were imaged using a Leica DMR microscope (Leica Microsystems) fitted with an RT-slider SPOT camera (Software version 4.0.4, Diagnostic Instruments).

\section{Quantification of CeA and BNST damage}

Electrolytic lesions were scored based on the extent of damage to the target and surrounding regions. The behavioral data presented here derive from males with lesions that damaged $>50 \%$ of the target tissue and $<50 \%$ percent of the tissue of any surrounding region. To illustrate lesions, sites of damage were traced onto images of a reference brain and stacked.

Unlike electrolytic lesions, the injection of IBO did not produce clearly visible tissue damage. To quantify the damage caused by both types of lesion in the same way, the number of cells remaining in target nuclei was assessed postmortem using Nissl-stained sections. The CeA was imaged bilaterally at three levels anterior to posterior (Moreno and González, 2005); the BNST was imaged at four levels (Moreno et al., 2012). Cells in each image were counted using ImageJ software (National Institutes of Health) with visual selection of threshold and watershed processing. Statistical analyses included the cell counts at each level of the CeA or BNST, as well as the total cell count from all levels.

\section{Behavioral analysis}

Behavioral data were not normally distributed with equal variances, so nonparametric statistics were used. To control for individual variation in baseline behavioral performance, changes in behavior $(\Delta)$ were calculated as [( postlesion score) - ( prelesion score) $]$ for each frog. Wilcoxon matched-pairs signed-rank tests were used for paired comparisons of behavior before and after surgery. To examine individual changes in time spent calling in the prestimulus, during stimulus, and poststimulus periods in playback tests, Friedman's test with Dunn's multiplecomparison post tests was used. The Kruskal-Wallis and Mann-Whitney $\mathrm{U}$ tests were used for unpaired data. Correlations between behavior and cell counts were examined with Pearson's product-moment correlation. For CeA lesions, IBO-lesioned and electrolytically lesioned animals were also grouped for correlation analysis. In all cases, changes in behavior correlated most strongly with the number of cells remaining in the most posterior aspect of the CeA or BNST, and we report those values. Analyses were performed using STATA (StataCorp), Prism (GraphPad), and $\mathrm{R}$ (R Foundation) software.

\section{Results}

\section{The CeA connects ascending auditory pathways to the VPG}

Social communication between $X$. laevis is predominantly acoustic, and interactions between conspecifics involve the evaluation of auditory cues to produce socially appropriate vocalizations. Previous research suggested that the ventral telencephalon is an important source of forebrain input to vocal-pattern generating circuitry (Brahic and Kelley, 2003). Nuclei within the ventral telencephalon, specifically the CeA and BNST, receive inputs from sensory thalamic nuclei (Marín et al., 1998b) that may include auditory information. This pattern of connectivity suggests that the CeA and BNST may play important roles in sensory motor integration, required for vocal communication. The general patterns of efferent and afferent connectivity of both the CeA and BNST have been described for multiple anuran species, including X. laevis (Marín et al., 1998a, b; Brahic and Kelley, 2003; Moreno and González, 2005; Moreno et al., 2012). Here, we focus on elucidating the details of their specific connectivity with nuclei that are known components of auditory and vocal circuits.

In anurans, CeA and BNST are located within the caudal-most portion of the ventral telencephalon, on either side of the caudal pole of the forebrain ventricle (Fig. 2A). Each nucleus is associated with one of the major efferent pathways of the anuran forebrain, CeA with the LFB and BNST with the stria terminalis (ST; Fig. 2A) (Moreno and González, 2005, Moreno et al., 2012). Small injections of tracer within the cell body layers of CeA and BNST show that CeA dendrites extend laterally through the LFB, 
whereas BNST dendrites are oriented toward the midline, passing through the ST and avoiding the LFB (illustrated in Fig. 2A; CeA, Fig. 2E; BNST, data not shown), and descending axons from each nucleus are similarly segregated within their respective efferent pathways.

\section{CeA connectivity}

The most prominent input to the CeA, based on retrogradely labeled cells after tracer injection, arises from the CT (Figs. 2C and $3 A-C$ ). The CT projection to the CeA outlines the perimeter of that nucleus, suggesting that CeA and the more rostrally located striatum are the major telencephalic recipients of sensory inputs from the multisensory CT with little, if any, projection to more dorsally located pallial derivatives, such as lateral amygdala (Fig. 2B,D,F). Although some previous reports have suggested that the region defined as CeA may represent a mixed population of amygdalar and pallidal homologs (Laberge et al., 2006), our results suggest instead that these populations are distinct. Using tracer injection into CT and visualization in both horizontal sections and in whole mount, we show that the CeA can be clearly distinguished from the adjacent, but more caudally located, entopeduncular area (EA; homolog of the pallidum). Injections into CT reveal back-labeled cells within the EA, indicative of a reciprocal connection with CT, but none in the CeA (Fig. 2B,D). $\mathrm{CT}$ injections also reveal afferent projections that travel within the LFB into the telencephalon, spreading across the lateral and ventral aspects of the subpallium, including striatum, and CeA (Fig. $2 B$ ). Anterogradely labeled CT axons pass through the dendritic fields of CeA cells (Fig. 2E,F).

Double-labeling of CeA and CT reveals putative contact between LFB axons arising from CT and dendritic spines of CeA neurons (Fig. 2E). These contacts are restricted to distal portions of the dendrites, avoiding the cell body layer and proximal-most third of the dendritic tree (Fig. 2E,F). Synaptophysin-positive puncta (strongly indicative of presynaptic specializations) (Spiwoks-Becker et al., 2001; Valtorta et al., 2004) are common within the axons comprising the LFB in the area that overlaps with the dendritic fields of CeA neurons. The density of puncta within this region was 7.8 per $100 \mu \mathrm{m}^{2}$ (Fig. $2 G$ ), indicating that these CT axons are likely to make functional synapses within CeA, rather than simply being fibers of passage, which do not contain synaptophysin puncta. No signal was seen in adjacent brain sections not incubated with the primary antibody (density within CeA LFB, 0.03 puncta per $100 \mu \mathrm{m}^{2}$ ). Colocalization of synaptophysin-positive puncta with LFB axons specifically labeled by CT injection (mean, 6.1 puncta per $100 \mu \mathrm{m}$ of CT axon within the CeA; Fig. $2 G$ ) supports the presence of synaptic contacts between CT and CeA cells.

The CeA has an important reciprocal connection with the hindbrain vocal center DTAM, as revealed by tracer injection into either nucleus (Figs. $2 D$ and $3 D-F$ ). This connection distinguishes the boundary of the CeA from the more rostrally located striatum, which has neither back-labeled cells nor terminal fields after DTAM injection (Fig. 2D). The terminal field arising from a DTAM tracer injection ramifies within the cell body layer and proximal dendrites of CeA cells and is distinct from the sensory axons arising from CT (Fig. 2D,F). EA cells are also not backlabeled by tracer injection into DTAM (Fig. 2D), again clearly demonstrating that the $\mathrm{CeA}$ is distinct from this pallidal homolog in terms of its gross connectivity.

Unlike the CeA, the BNST does not receive input from CT (Fig. $2 B, D, F$ ) and makes no direct afferent or efferent connections with DTAM (Fig. $2 F$ ). The BNST does receive a projection from $\mathrm{CeA}$ through the anterior commissure, and CeA also projects across hemispheres to the other CeA via this fiber pathway. The connection between CeA and BNST is not reciprocal (Fig. 2H) (Moreno and González, 2005, Moreno et al., 2012).

\section{CT connects CeA to auditory circuits}

The major input to the CT arises from the midbrain IC (Edwards and Kelley, 2001). Tracer injection into CT labels cells within each subdivision of the IC, including the auditory laminar nucleus (data not shown). Tracer injection into either the laminar or principal subdivisions of the IC labels thin diameter axons within the CT with many varicosities, characteristic of a terminal field (Fig. 3A-C) (Edwards and Kelley, 2001). The terminal field arising from the IC overlaps with the population of thalamic cells labeled by injections into CeA (Fig. 3B,C; magenta terminals, green dendrites). When the proximal dendrites and the somata of CT cells are labeled, instances of putative synaptic connections between axon boutons arising from the IC (magenta) and thalamic dendrites (green) can be identified in confocal optical sections from double-labeled brains (Fig. $3 C$ ), indicating that CT inputs to CeA are likely to include auditory information from the IC.

\section{CeA is connected with vocal circuits}

To confirm that the connectivity between CeA and DTAM in X. laevis is relevant to vocal and respiratory circuits, we performed dual tracer injections into both CeA and the VMN. VMN injections retrogradely label large cells in DTAM that lie within the terminal field arising from CeA injection (Fig. $3 D-F$ ). In doublelabeled brains, putative synaptic boutons labeled by CeA injection were frequently observed making contacts with the somas and proximal dendrites of these VMN-projecting cells (Fig. $3 E, F)$. Synaptophysin-positive puncta are common on CeA axons within the boundaries of DTAM (21.4 per $100 \mu \mathrm{m}$ of CeA axon within DTAM), again indicating that CeA axons within DTAM represent a true monosynaptic connection rather than fibers of passage. Direct input from CeA to the vocal premotor nucleus DTAM suggests that CeA is capable of influencing activity within vocal-respiratory circuits to modulate vocal behavior.

\section{The effects of stimulation on fictive vocal behavior ex vivo}

The anatomical connections between the auditory system and the VPG via the CeA suggest that telencephalic activity is capable of modulating vocal activity, perhaps, for example, influencing the decision to vocalize or the production of different call types. We examined this hypothesis in an isolated brain preparation by driving CeA with extracellular current injection while recording from the laryngeal nerve, predicting that CeA stimulation would influence VPG activity as measured by a change in nerve activity. Because neither the patterns of telencephalic input to the VPG nor the auditory cues that drive CeA neurons are known, we used multiple stimulation paradigms to explore this connection.

We applied short pulse $(0.1-0.5 \mathrm{~ms})$ stimulus trains to the CeA and other sites within the telencephalon (Fig. $4 A$ ), which produced laryngeal nerve activity ranging from desynchronized bursts of activity with a short latency to fictive advertisement calls with a biphasic CAP pattern similar to in vivo calling. On average, $73 \%$ of short pulse stimuli delivered to the CeA produced desynchronized bursts of activity recorded from the laryngeal nerve, a value significantly different from all other stimulated telencephalic areas (Mann-Whitney U: $z=2.2, p=0.0278$ ). CeA stimulation induced bursts at a shorter latency (average $787 \mathrm{~ms}$ ) than the bursts induced by stimulation of other regions (Mann-Whit- 


\section{A Stimulation Sites within the Telencephalon}

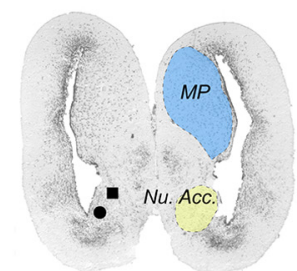

i

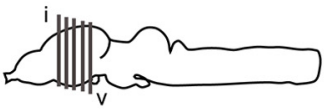

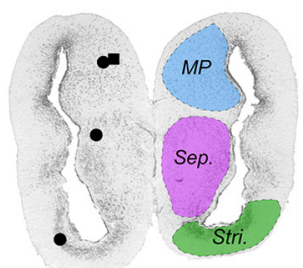

ii

- Short pulse: Fictive call

- Short pulse: No call

iii
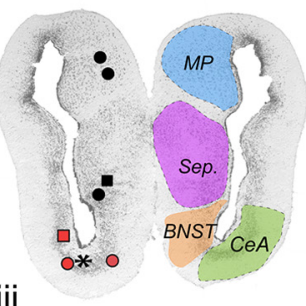

iv

$\square \quad$ Long pulse: Fictive call

- Long pulse: No Call

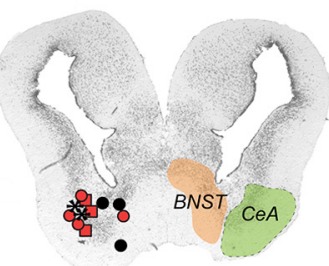

* Called to Lesion Current Pulse

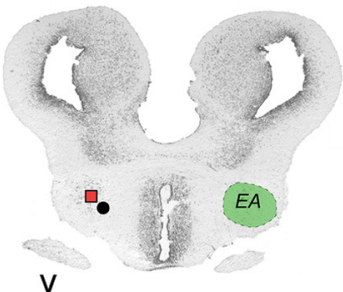

V

\section{B 5-HT Application}

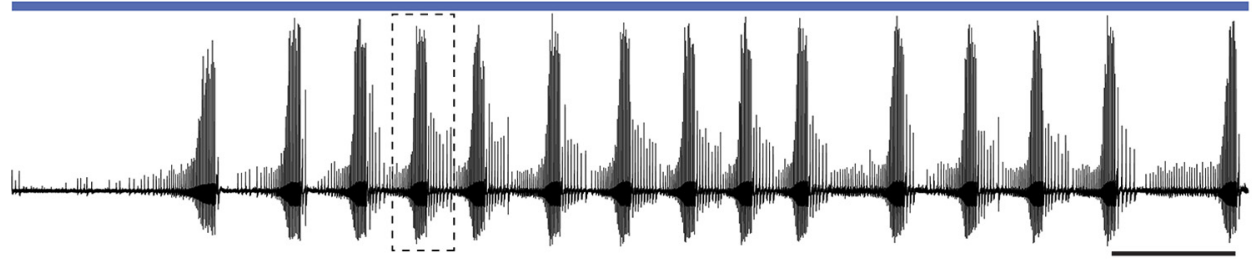

C Short Pulse Stimulation

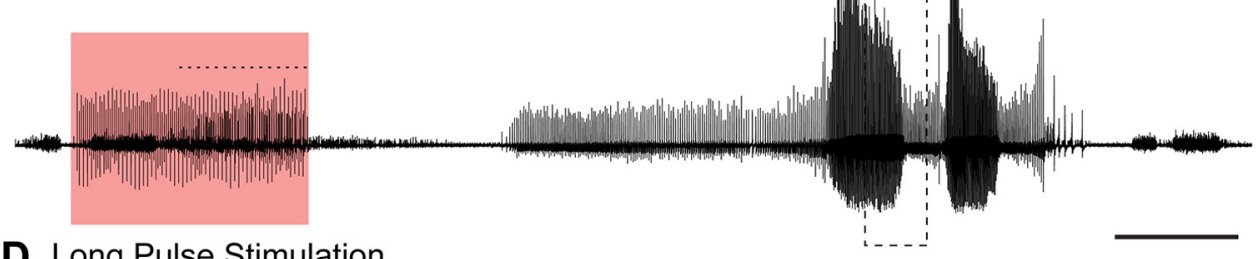

D Long Pulse Stimulation

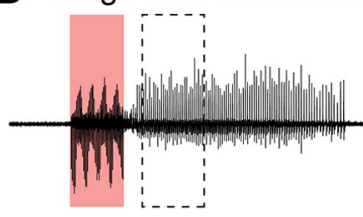

E CAP Shape

Raw Traces

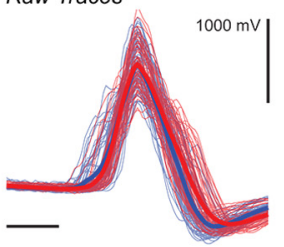

Normalised to Peak

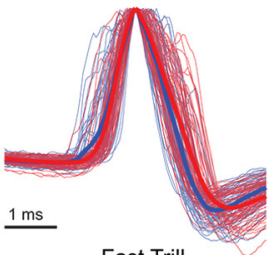

Fast Trill
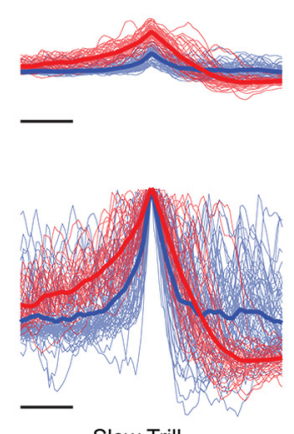

F CAP Dimensions

Fast Trill CAPs

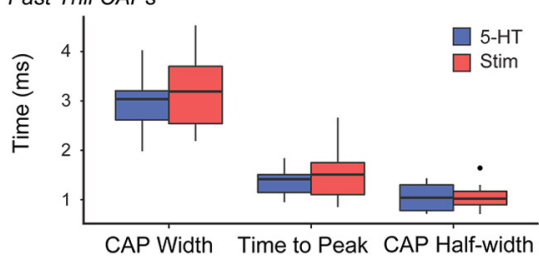

Slow Trill CAPS

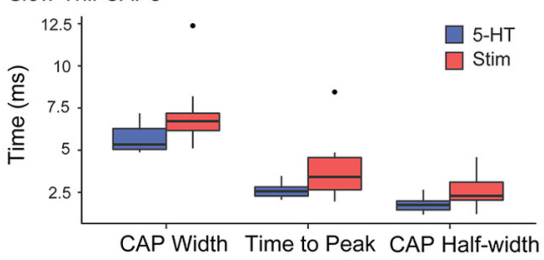

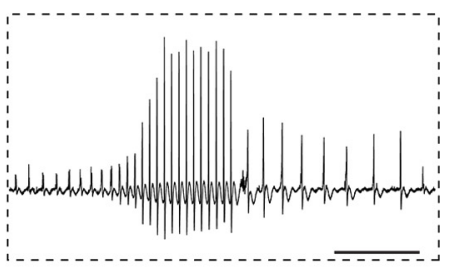
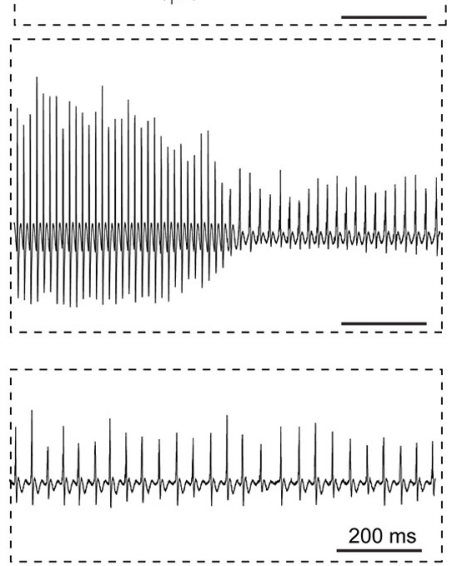

$2 \mathrm{~s}$

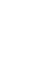


Table 1. A summary of the effects of long and short pulse stimulation of areas in the forebrain on laryngeal nerve activity ${ }^{a}$

\begin{tabular}{|c|c|c|c|c|c|c|c|c|}
\hline \multicolumn{3}{|l|}{ Brain region } & \multirow{3}{*}{$\begin{array}{l}\text { Average } \% \text { of stimulus } \\
\text { trains producing: } \\
\text { Desynchronized activity }\end{array}$} & \multicolumn{3}{|c|}{ Desynchronized activity (ms) } & \multirow{2}{*}{\multicolumn{2}{|c|}{ Fictive calling (ms) }} \\
\hline & & Average number of & & \multirow{2}{*}{$\begin{array}{l}\text { Fictive } \\
\text { calling }\end{array}$} & \multirow[b]{2}{*}{ Latency } & \multirow[b]{2}{*}{ Duration } & & \\
\hline & $n$ & stimulus trains & & & & & Latency & Duration \\
\hline \multicolumn{9}{|l|}{ Long pulse stimulation } \\
\hline CeA & 4 & $20(5)$ & $75(24)$ & $31(22)$ & $90(10)$ & 6400 (1199) & $13,756(14,781)$ & $21,218(15,744)$ \\
\hline EA & 1 & 20 & 95 & 15 & 61 & 7573 & 14926 & 4907 \\
\hline Nucleus accumbens & 1 & 40 & 13 & 0 & 2547 & 3563 & NA & NA \\
\hline Septum & 1 & 37 & 53 & 0 & 1188 & 2805 & NA & NA \\
\hline Pallium & 1 & 11 & 64 & 0 & 152 & 4218 & NA & NA \\
\hline \multicolumn{9}{|l|}{ Short pulse stimulation } \\
\hline CeA & 8 & $37(13)$ & $73(23)$ & $5(9)$ & 787 (1225) & $10,323(12,515)$ & $29,928(21,129)$ & $7432(6760)$ \\
\hline BNST & 4 & $47(5)$ & $45(30)$ & $2(3)$ & $1803(637)$ & $2683(1740)$ & $48,646(28,225)$ & $7594(6065)$ \\
\hline EA & 1 & 27 & 81 & 0 & 95 & 7477 & NA & NA \\
\hline Nucleus accumbens & 1 & 28 & 43 & 0 & 516 & 4795 & NA & NA \\
\hline Septum & 2 & 37 (1) & $45(22)$ & $0(0)$ & 1367 (791) & $4039(2263)$ & NA & NA \\
\hline Pallium & 3 & $34(7)$ & $45(20)$ & $0(0)$ & 1102 (1026) & 6055 (2176) & NA & NA \\
\hline Striatum & 1 & 40 & 0 & 0 & NA & NA & NA & NA \\
\hline
\end{tabular}

${ }^{a}$ Data are mean $\pm S D$, reported for the number, latency, and duration of bouts of desynchronized bursting activity and fictive calling episodes. The effects of telencephalic stimulation on fictive calling and bursting activity in the laryngeal nerve. NA, Not applicable.

ney $\mathrm{U}: z=-2.132, p=0.033$; Table 1 ) and CeA bursts were of long duration (average 10,323 ms; Table 1). Stimulation of the EA also produced bursts of activity with a pattern similar to CeA stimulation (Table 1). Because CeA axons course through the EA toward DTAM, we were unable to determine whether activity after EA stimulation is a direct result of EA stimulation or due to recruitment of fibers of passage from the CeA. We have thus excluded the EA from our analyses.

When stimulating the CeA or the adjacent BNST, desynchronized activity was sometimes accompanied by fictive calling. After stimulation, 6 of 20 male brains produced biphasic and monophasic CAP trains comparable in temporal structure to

$\leftarrow$

(Figure legend continued.) (indicated by the red box, long pulse: $2 \mathrm{~ms}$ duration, $60 \mathrm{~Hz}$ ) shows two monophasic trills after stimulation: one immediately after the cessation of the stimulus train (expanded time-scale of the dashed region shown in inset) and the second $\sim 5 \mathrm{~s}$ later. Calibration: $\boldsymbol{D}, 2 \mathrm{~s}$; inset, $200 \mathrm{~ms}$. $\boldsymbol{E}$, Individual CAPs elicited by $5-\mathrm{HT}$ and electrical stimulation (stim) from the same brain. Fast trill CAPs have similar shapes and heights regardless of mode of stimulation, whereas slow trill CAPs are less stereotyped both within and between modes of stimulation. Individual CAPs were extracted from 1- to 2-s-long stretches of fictive calling activity for periods of both $5-\mathrm{HT}$ and electrically stimulated fictive calling in a single brain (stimulation CAPs in this example come from the trace shown in $\boldsymbol{B}$ ). Plots represent 5 -ms-long stretches of N.IX-X nerve activity centered on the peak of the CAP with individual CAPs overlaid. All CAPs originated from periods of alternating biphasic calling, but only those that could be reliably assigned to fast or slow trills were used for this plot. Upper row, Raw CAP traces separated into fast and slow trill groups and color-coded by the mode of stimulation that elicited them. Fast and slow CAPs are both plotted with the same vertical scale (vertical scale bar represents $1000 \mathrm{mV}$ ). Thicker lines represent the mean across all individual CAPs of that type represented on this plot. Lower row, Same CAPs normalized by peak height for both fast and slow trills, again color-coded by the mode of stimulation, with bold lines representing the mean of all CAPs in each group. Horizontal scale bar for each plot represents $1 \mathrm{~ms}$. F, CAP width, time to peak, and CAP half-width displayed as box-and-whisker plots for fast trill and slow trill CAPs elicited by 5 -HT and electrical stimulation. Measurements from 25 instances of each type of CAP (fast and slow for 5-HT and stim-elicited episodes) were averaged to produce a single value for each type of CAP for each brain that produced fictive calling episodes to both modes of stimulation. Upper plots, Values for fast trill CAPs; lower plots, those for slow; both plots, color coded for mode of stimulation. Thick line indicates the median across all brains; box represents the upper and lower quartiles, with tails showing the upper and lower extremes. Black dots indicate outliers ( $>3$ SD above median). G, ICI histograms showing the bimodal distribution of in vivo and fictive calling. All measured clicks (in vivo) and CAPs (fictive) were pooled across brains and calling episodes, and in aggregate show bimodal distributions with a "fast" peak centered at $\sim 16 \mathrm{~ms}$ and a broader "slow" peak whose mean ICl is more variable between conditions. The $n$ value indicates the number of frogs or brains that data were pooled from for each condition. calls or call segments: these trains were designated as episodes of fictive calling. Histological analysis indicated that 4 of 6 sites that produced fictive calling episodes were located within the CeA (4 of 8 sites that were located in this nucleus), either within the cell body layer or immediately adjacent to it in the vicinity of the proximal dendrites (Fig. $4 A$, red circles). The remaining two positive sites were located within the proximal dendritic layer of BNST ( 2 of 4 sites within BNST). There were no systematic differences between the stimulus rate or the duration of short pulse trains that produced fictive calling and those that were ineffective (Mann-Whitney U: rate, $z=1.104, p=0.2696$; duration, $z=$ $0.226, p=8209$ ). Stimulation of 11 other sites within the EA, septum, medial pallium, striatum, and nucleus accumbens never elicited fictive calling (Fig. 4A, black circles; Table 1). The short pulse stimulus trains used at nonfictive calling sites differed somewhat in average rate ( $38 \mathrm{vs} 29 \mathrm{~Hz}$, Student's $t$ test: -4.6356 , $p<0.001)$ and duration (3218 vs $4469 \mathrm{~ms}$, Student's $t$ test: $5.3415, p<0.001$ ) from the stimuli used at sites that produced fictive calling. Although this was not intentional, stimulation of control sites was both shorter duration and higher frequency than of sites within CeA. There was, however, no difference in the number of pulses applied to control and CeA sites (Student's $t$ test: $p=0.88$ ).

Within the six brains that produced fictive calling episodes after stimulation of the telencephalon, on average $8 \%$ (range, $2-22 \%$; SD, 7\%) of stimulation events were followed by an episode of fictive calling. A total of $38 \%$ of these episodes included alternating biphasic CAP trains whose temporal patterns closely resembled those produced by 5 -HT application (Fig. $4 C, F$ ). In the remaining $62 \%$, short pulse stimulation-evoked fictive calling consisted of monophasic CAP trains of variable duration (range, 0.56-18.6 s; median, $3.1 \mathrm{~s}$ ), with ICIs comparable with either the fast or slow trill of the advertisement call (compare Fig. $4 E$, $F$ with Fig. 1A; Table 2). Latencies to fictive call production after stimulation varied but were significantly shorter on average than the latencies to fictive calling observed after 5-HT application (stimulation: range, 0.25-86.3 s; median, $12.2 \mathrm{~s}$; 5-HT: range, 8.9-128 s; median, 20.0 s; Kruskal-Wallis: $\mathrm{H}=20.429, p=0.0001)$.

Five brains were stimulated within CeA or BNST without eliciting any fictive calling episodes after short pulse trains. In 3 of these 5 brains in which the short pulse train stimulation $(0.1-0.5$ $\mathrm{ms}, 150 \mu \mathrm{A}$ individual pulses) in the CeA failed to elicit fictive 
Table 2. Advertisement and fictive advertisement calls are composed of a fast trill phase and a slow trill phase, distinguished by the rate of clicks or CAPs, respectively $^{a}$

\begin{tabular}{lll}
\hline Method of call induction & $\mu 1, \mathrm{~ms}$ & $\mu 2, \mathrm{~ms}$ \\
\hline In vivo & $16.6 \pm 2.0$ & $33.0 \pm 10.3$ \\
Long pulse stimulation & $15.8 \pm 2.0$ & $38.1 \pm 7.4$ \\
Short pulse stimulation & $14.1 \pm 1.8$ & $31.3 \pm 16.4$ \\
5-HT & $17.3 \pm 2.3$ & $44.4 \pm 23.6$
\end{tabular}

${ }^{a}$ Data are mean \pm SD of fictive and in vivo call ICI distributions, reported for in vivo interclick intervals and ex vivo ICls during fictive calls induced by electrical or chemical stimulation ex vivo.

calling, the $250 \mathrm{~ms}, 1.5 \mathrm{~mA}$ pulse that was used to lesion the stimulation site for histological analysis did elicit fictive calling episodes at short ( $<5 \mathrm{~s}$ ) latencies (Fig. $4 A$, asterisks). Each effective stimulation site was located within the boundaries of the $\mathrm{CeA}$. When the electrode was located outside of the CeA (including one case where it was located within the cell body layer of BNST; Fig. 4A, black circles), the same long duration, high current pulses used to produce lesions were never effective in eliciting fictive calling episodes. Electrode sites located within BNST but which failed to elicit fictive calling episodes to either short duration pulse trains or high intensity current pulses were situated dorsally in the nucleus (Fig. 4Aiii,iv, BNST shown in orange). In the two brains in which BNST stimulation did elicit fictive calling, the electrode was located more ventrally, within the layer containing the commissural axons that connect CeA across the two hemispheres.

Because the very intense electrical stimulation used to lesion stimulation sites produced fictive calling responses in brains that failed to respond to less intense stimulation, we explored the possibility that intermediate levels of stimulation would be more successful. We thus stimulated 8 brains with trains of pulses of the same intensity as our short pulse trains $(150 \mu \mathrm{A})$ but of longer duration (1-2 ms). This stimulation paradigm (long pulse) delivers $\sim 10$ times the charge per pulse of the short pulse trains (the lesion pulses delivered $\sim 20,000$ times greater charge). Long pulse stimulation (1-2 ms per pulse) was effective at eliciting fictive calls from 5 of these 8 brains. Four of 5 stimulation sites that successfully elicited calling after long pulse stimulation were located within $\mathrm{CeA}$, whereas the fifth was located within EA, where the LFB exits the telencephalon (Fig. $4 A$, red squares). On average, $75 \%$ of long pulse stimulation events in the CeA were followed by desynchronized bursts of laryngeal nerve activity with an average latency of $90 \mathrm{~ms}$, shorter than those induced by stimulation of regions that did not produce fictive calling (Table 1; Mann-Whitney $\mathrm{U}: z=-2.121, p=0.0399)$. The average duration of desynchronized laryngeal nerve activity was $6400 \mathrm{~ms}$. A total of 27\% (range, 7-59\%; SD, 20\%) of long pulse stimulus trains in these brains were followed by fictive calling, with variable latency and duration (latency: range, 0.59-48.0 s; median, $1.0 \mathrm{~s}$; duration: range, $0.46-131.7 \mathrm{~s}$; Fig. 4D; Table 1). More rapid stimulus trains were more effective at evoking fictive calling than slower trains (average, 46 vs $30 \mathrm{~Hz}$, Mann-Whitney $\mathrm{U}: z=$ $-3.021, p=0.0025)$.

Long pulse trains were more effective than short pulse trains in eliciting fictive calling (two-sample test of proportions: $z=$ $-2.42, p=0.016$ ). Monophasic fictive calling, either fast or slow trill segments, occurred with similar frequency in both stimulation paradigms. However, fictive calls that alternated between fast and slow trill phases were observed less frequently after long pulse stimulation than short pulse stimulation (mean: long pulse: 8.3\%; two-sample test of proportions: $z=2.01, p=0.045$ ). Long pulse stimulation of the medial pallium, nucleus accumbens, and septum was ineffective at eliciting fictive calling (Fig. $4 A$, black squares), despite being delivered at longer durations than those that produced fictive calling elsewhere (5026 vs $886 \mathrm{~ms}$, Student's $t=-17.54, p<0.001)$.

When ICIs from all stimulation-elicited fictive calling episodes (monophasic and biphasic) were pooled, the resulting distributions were clearly bimodal and closely resemble the distribution of interclick intervals measured from advertisement calling in vivo (Fig. 4G). We fitted mixed Gaussians to the ICI distributions for fictive calling elicited by both 5-HT and electrical stimulation, and in vivo advertisement calling, and obtained comparable values across the four conditions for $\mu 1$, representing in vivo fast trill (Fig. 4G; Table 1). $\mu 2$, representing in vivo slow trill, was more variable both between and within conditions (electrical and 5-HT stimulation), as has been reported previously (Rhodes et al., 2007; Zornik et al., 2010, Zornik and Yamaguchi, 2012).

In vivo, CAP shapes vary with call type and between fast and slow trill (Yamaguchi and Kelley, 2000). These differences are also apparent in CAPs comprising 5-HT elicited fictive calling ex vivo (Rhodes et al., 2007). To determine whether electrically evoked activation of vocal motor neurons matched activity during singing at a scale finer than just their temporal patterns, we compared CAP shapes of fast trill elicited by electrical stimulation of the forebrain with those elicited by 5-HT application. For each brain that produced at least one bout of fictive calling after forebrain stimulation, we measured the shapes of a subset of the CAPs elicited by each condition. The shapes of fast trill CAPs elicited by current injection in the telencephalon were similar to those elicited by bath application of 5-HT (Fig. 4E,F). Fast trill CAPs elicited by current injection did not differ from those elicited by 5 -HT in the same brain in width (paired $t$ test: $t=-1.040, p=$ 0.33 ), time to peak (paired $t$ test: $t=-0.539, p=0.60$ ), or half-width (paired $t$ test: $t=-0.065, p=0.95$; Fig. $4 F$ ), indicating that fast trill CAPs elicited by current injection are likely generated by the same mechanism within the VPG as those elicited by 5 -HT.

Interestingly, the peak heights of fast trill CAPs elicited by current injection were significantly smaller than those of 5-HTelicited fast CAPs (paired $t$ test: $t=5.934, p<0.0005$ ). CAP heights reflect the number of motor neurons that are simultaneously active. That electrically evoked CAPs have smaller peak heights than 5-HT-evoked CAPs without an increase in CAP duration suggests reduced recruitment of motor neurons, rather than reduced synchrony across the active population. This result is consistent with the idea that, although current injection activates the same vocal-pattern generating circuitry as bath-applied 5 -HT, it activates a smaller proportion of the neurons within the circuit. That 5-HT application elicits fictive calling in a larger percentage of preparations may reflect the global and sustained nature of its application as opposed to the more focal and transient excitation provided by electrical stimulation. Slow trill CAPs elicited by current injection did not significantly differ from those elicited by 5 -HT in terms of CAP height (paired $t$ test, $t=-2.204, p=0.063$ ), width (paired $t$ test, $t=-2.205, p=$ 0.063 ), half-width (paired $t$ test, $t=-2.218, p=0.062$ ), or time to peak (paired $t$ test, $t=-1.916, p=0.097$ ), although trends in each of these measurements toward larger values for current injection CAPs were observed (Fig. 4E, upper right panel, $F$, lower row). The trend for electrically induced fictive CAPs to be slightly higher and wider may simply be a result of intrinsically higher variability in slow trill CAPs as opposed to fast trill CAPs, rather 
than an indication of any underlying difference between the generation of slow trill CAPs by the two types of stimulation.

\section{The effects of CeA lesions in vivo on behavior}

The connection between the ascending auditory pathway and the VPG through the CeA and the observation of fictive calling after stimulation of the CeA ex vivo suggested that the CeA could play a role in the modulation of vocal activity in response to acoustic cues. To examine this hypothesis, we damaged the CeA with excitotoxic and electrolytic lesions and examined the effects of these manipulations on responses to playbacks of social vocalizations. The behavior of CeA-lesioned males was compared with their presurgical values and the behavior of males that underwent a sham lesion.

The behavior of sham-lesioned males in response to playbacks of social vocalizations was consistent with previously published results in intact frogs. During playbacks of male advertisement calling, sham-lesioned males demonstrated prolonged vocal suppression (Freidman $\mathrm{Q}=14.11, p<0.001$ ); they ceased calling a few seconds into the playback (rapid suppression) and $78 \%$ did not resume calling until the $900 \mathrm{~s}$ playback was over (Fig. 5A). During playbacks of female rapping calls, which communicate readiness to mate, sham-lesioned males called throughout, or only briefly met suppression criteria (Fig. $5 A$ ) and increased their overall rate of calling (Friedman $\mathrm{Q}=10.89, p=0.0029$ ). In response to female ticking, which communicates nonreceptivity, sham-lesioned males responded with transient vocal suppression (Friedman $\mathrm{Q}=11.56, p=0.0013$ ), ceasing calling at the onset of the ticking playback and resuming calling $187 \mathrm{~s}$, on average, after suppression. Sham-lesioned males differed in the time to suppression for all three calls (Fig. $5 \mathrm{~A}$, star; Friedman Q = $6.353, p=0.0307$, Dunn's multiple comparison) and for resumption of calling (Fig. 5A, star; Friedman $\mathrm{Q}=9.172, p=$ 0.006, Dunn's multiple comparison).

Bilateral lesions of the CeA with IBO injection $(n=9)$ produced alterations in male responses to broadcasts of social vocalizations (Fig. 5B). After IBO lesion, males still demonstrated prolonged suppression to playbacks of male advertisement calls (Friedman: $\mathrm{Q}=8.222, p=0.0158$ ). However, IBO-lesioned males did not increase their rate of calling during playbacks of female rapping (Friedman: $\mathrm{Q}=2.889, p=0.2781$ ), nor did they decrease calling during playbacks of female ticking (Friedman: $\mathrm{Q}=0.222, p=0.9712$.

Bilateral electrolytic lesions in the CeA $(n=12$; Fig. $6 A)$ had more pronounced effects. Electrolytically lesioned males responded to all three playbacks with decreased vocalization rates (Fig. 5B; Friedman: advertisement, $\mathrm{Q}=15.80, p<0.001$; rapping, $\mathrm{Q}=7.128, p=0.0303$; ticking, $\mathrm{Q}=9.8, p=0.0063)$. The most frequent response to playbacks ( $91 \%$ of advertisement responses, $58 \%$ of rapping, $66 \%$ of ticking) was rapid and sustained vocal suppression, in which calling resumes only after the playback is over. Persistent suppression to rapping playbacks was observed significantly more often in electrolytically lesioned males than in sham-lesioned males (two-sample test of proportions, $z=-2.55, p=0.011$ ).

Unlike sham-lesioned males, CeA-lesioned males showed no difference in the time to suppression (Friedman, IBO: $\mathrm{Q}=$ $6.0, p=0.057$; electrolytic: $\mathrm{Q}=3.211, p=0.1873$ ) or time to resumption of calling (Friedman, IBO: $\mathrm{Q}=0.9630, p=$ 0.5690 ; electrolytic: $\mathrm{Q}=3.0, p=0.2223)$ to playbacks of advertisement, rapping, or ticking calls (Fig. 5B). The time to resumption of calling during rapping playbacks was significantly greater after CeA lesions than sham lesions, and this
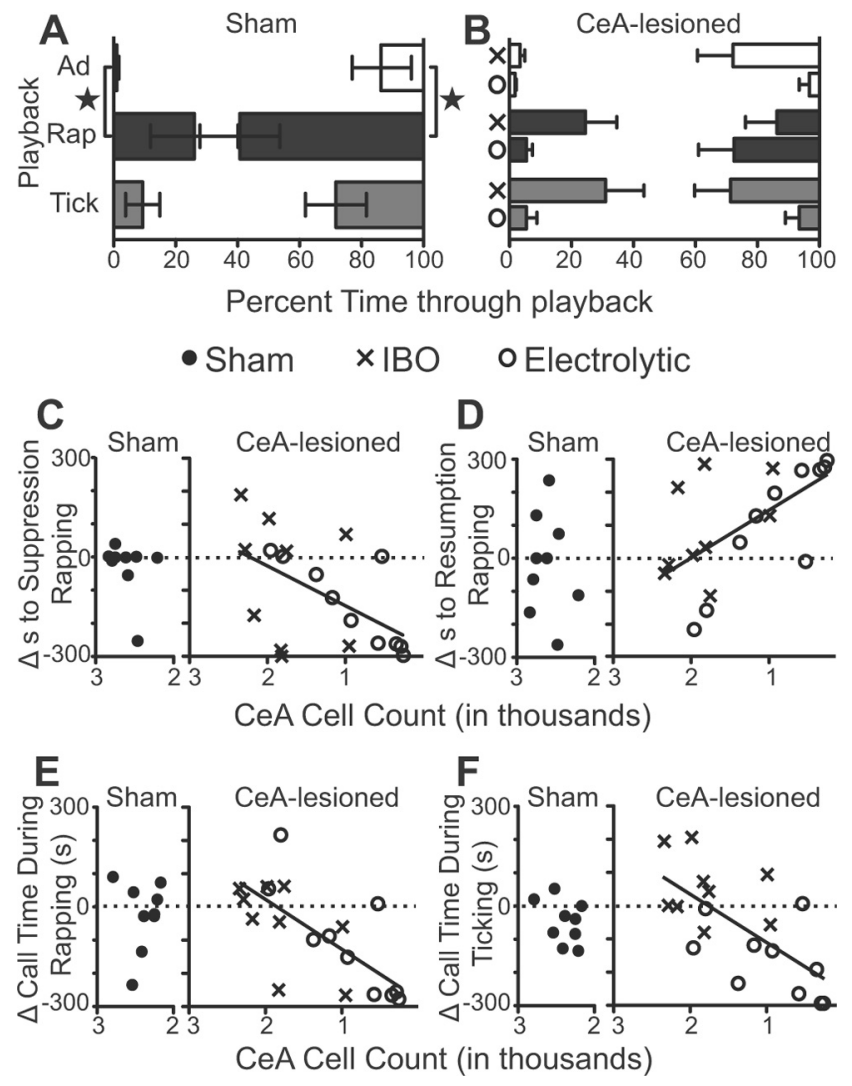

Figure 5. The response to playbacks was altered by CeA lesion. $\boldsymbol{A}$, The time to suppression of calling (left $y$-axis) and the time to the resumption of calling (right $y$-axis) during playback are plotted for advertisement (Ad), rapping, and ticking. Sham-lesioned males suppressed earlier and resumed calling later during advertisement playbacks than rap playbacks (stars; Friedman, Dunn's multiple comparison, $Q=6.5, p=0.03$ for suppression; $Q=9.2, p=0.006$ for resumption). Advertisement playbacks were $15 \mathrm{~min}$ long; rap and tick playbacks were $5 \mathrm{~min}$ long, so that the $X$-axis is normalized for the duration of each playback. $\boldsymbol{B}$, Using the same conventions as in $\boldsymbol{A}$, CeA-lesioned animals show prolonged vocal suppression during advertisement, rap, and ticking playbacks. After IBO or electrolytic lesion, there was no significant difference in the time to suppression or resumption of calling during any playback (suppression: Friedman, IBO, $Q=6.0, p=0.057$; electrolytic, $Q=3.211, p=0.1873$; resumption: Friedman, $I B 0, Q=0.9630, p=0.5690$; electrolytic, $Q=3.0, p=0.2223)$. $C, D$, The prelesion to postlesion change in time to suppression ( $\boldsymbol{C}$ and time to resumption $(\boldsymbol{D})$ was correlated with the number of cells remaining in the posterior CeA after lesion. The $x$-axes are reversed, with higher cell counts on the left and lower on the right. The $y$-axis was calculated as ([post lesion behavior] - [prelesion behavior]), so positive numbers indicate greater postlesion values. The fewer cells remaining in the $C \mathrm{~A}$, the more rapid the time to calling suppression (C, Pearson's $r=0.54, p=$ 0.020 ) and the longer the time until call resumption ( $\boldsymbol{D}$, Pearson's $r=-0.62, p=0.01) . \boldsymbol{E}, \boldsymbol{F}$, The prelesion to postlesion change in the duration of calling during rapping $(\boldsymbol{E})$ and ticking $(\boldsymbol{F})$ playbacks was correlated with the number of cells remaining in the posterior CeA after lesion. The fewer cells remaining in the $(e A$, the less animals called during rapping ( $\boldsymbol{E}$, Pearson's $r=$ $0.69, p=0.001)$ or ticking $(\boldsymbol{F}$, Pearson's $r=0.68, p=0.001)$.

effect was most pronounced when IBO was used to lesion the CeA (Kruskal-Wallis, Dunn's multiple comparisons: $\mathrm{H}=$ 6.269, $p=0.0435$, Fig. 5B).

Alterations in male vocal responses to playbacks of female vocalizations were correlated with the extent of damage to the CeA. The cell counts from the IBO-lesion group were greater than those of the electrolytic-lesion group (Student's $t$ test, $p=$ 0.0017 ), suggesting that relatively more neurons were spared after excitotoxin application. To examine correlations between the extent of damage to the CeA and changes in behavior, vocal suppression and resumption of calling for individual frogs were compared with the number of CeA cells remaining after lesion. 


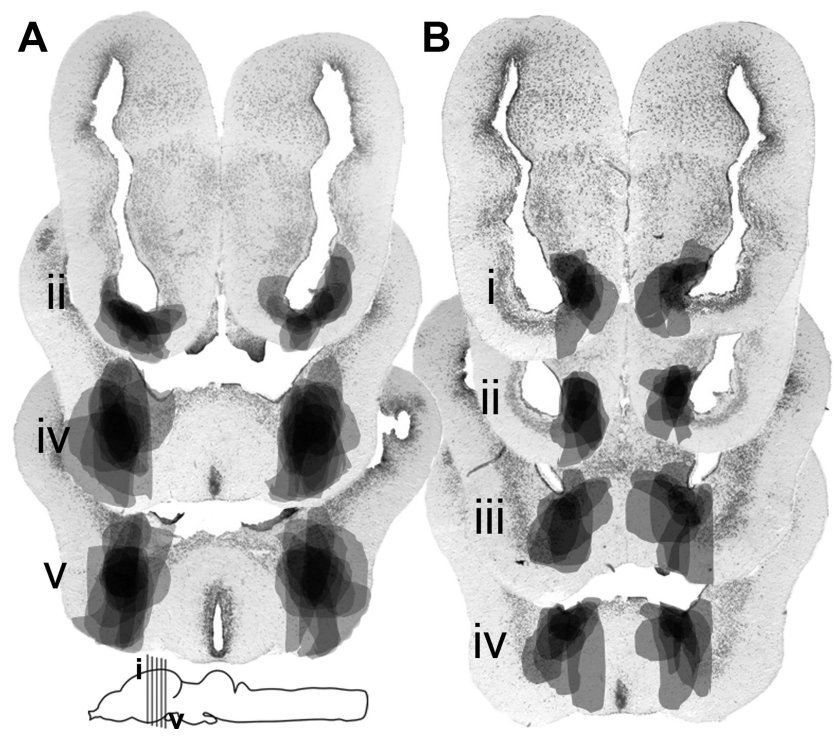

Figure 6. Transverse sections through the $X$. laevis forebrain at the level of the CeA and BNST. Electrolytic lesion sites for $(\boldsymbol{A})$ the CeA $(n=12)$ and $(\boldsymbol{B})$ the BNST $(n=9)$ were traced, colored gray, and overlain. Inset, Schematic of a sagittal view of the frog brain (rostral left) with vertical lines indicating the approximate level of the sections.

Frogs with fewer cells remaining in the CeA after lesion suppressed more rapidly to rapping (Pearson's $r=0.54, p=0.020$; Fig. $5 C$ ) or ticking (Pearson's $r=0.62, p=0.004$, data not shown), and took longer to resume calling than they did before lesion (rapping, Pearson's $r=-0.62, p=0.01$; Fig. $5 D$; ticking, $r=-0.57, p=0.01)$. Suppressing vocalization sooner and resuming calling later contributed to an overall correlation between fewer cells in the CeA and less calling during rapping and ticking playbacks (rapping, Pearson's $r=0.68, p=0.001$; ticking, $r=$ $0.69, p=0.001)$. Moreover, frogs with fewer cells remaining in the CeA called less than their presurgical values during playbacks of rapping (Pearson's $r=0.69, p=0.001$; Fig. $5 E$ ) or ticking (Pearson's $r=0.68, p=0.001$; Fig. $5 F$ ).

Although $X$. laevis change their vocalization patterns in response to playbacks of acoustic communication calls, some vocalizations are produced more reliably, or only, in the presence of a conspecific. Lesioned animals were thus allowed to interact with an actual female or male and vocalizations recorded. The rate of calling during a $10 \mathrm{~min}$ interaction with a male or female did not differ before and after CeA lesion (Wilcoxon, IBO: male, $p=$ 0.4961; female, $p=0.4609$; electrolytic: male, $p=0.0674$; female, $p=0.9593)$, or between sham- and CeA-lesioned males (Kruskal-Wallis: male, $\mathrm{H}=1.622, p=0.4444$; female, $\mathrm{H}=$ $0.5363, p=0.7648)$. However, CeA-lesion resulted in decreased production of a specific call type, the answer call, an intense form of the advertisement call with a longer fast trill segment and a shorter slow trill segment given during vocal interactions with conspecifics (Tobias et al., 1998). After electrolytic lesion of the $\mathrm{CeA}$, males produced fewer answer calls than sham-lesioned males when paired with a male (Kruskal-Wallis, Dunn's multiple comparisons, $\mathrm{H}=13.05, p=0.002$; Fig. $7 A$ ), or with a female (Kruskal-Wallis, Dunn's multiple comparisons, $\mathrm{H}=13.05, p=$ 0.002; Fig. 7C). When the IBO-lesioned and electrolytically lesioned groups were pooled, the number of cells remaining in the CeA was positively correlated with the number of answer calls directed at another frog; the fewer the cells remaining in the CeA after lesion, the greater the decrease in answer calls directed at a male (Pearson's $r=0.39, p=0.046$; Fig. $7 B$ ) or female (Pearson's
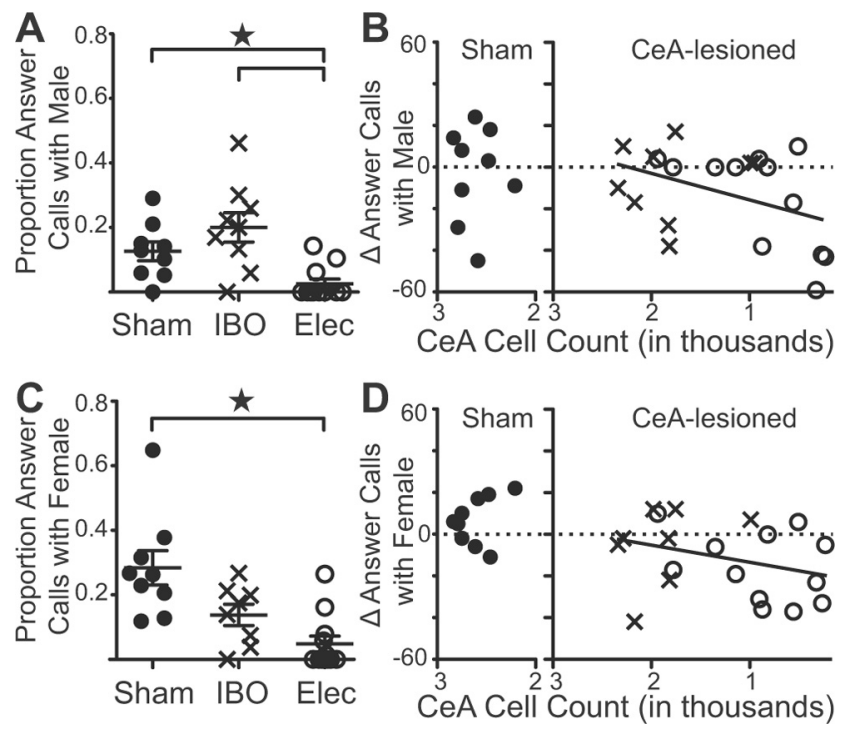

Figure 7. Answer call production decreases after CeA lesion. $\boldsymbol{A}$, The proportion of answer calls to all calls produced by a lesioned frog when paired with another male frog for 10 min was significantly reduced after electrolytic lesion of the CeA. Star and brackets indicate significantly different populations (Kruskal-Wallis $K=14.8, p=0.001$ ). $\boldsymbol{B}$, The prelesion to postlesion change in the number of answer calls produced during an interaction with a male frog was correlated with the number of cells remaining in the CeA (male: Pearson's $r=0.39, p=0.046$ ). C, The proportion of answer calls directed at a female frog during a 10 min interaction was significantly reduced after electrolytic lesion of the CeA (Kruskal-Wallis $\mathrm{K}=13.05, p=0.002$ ). $D$, The prelesion to postlesion change in the number of answer calls produced during an interaction with a female frog was correlated with the number of cells remaining in the CeA (Pearson's $r=0.44, p=0.026$ ).

$r=0.44, p=0.026$; Fig. $7 D)$. Thus, during tests with a male or female frog, damage to the CeA was correlated with a deficit in the production of the specific call type that would normally be evoked in this social context, but not with the overall amount of time spent calling.

\section{The effects of BNST lesions in vivo on behavior}

Although there are no direct projections from the BNST to the VPG, stimulation of the BNST ex vivo induced fictive calling. It is unclear whether fictive calling in the ex vivo prep represents direct activation of BNST neurons that increases activity within the VPG via indirect connections or whether fictive vocal responses after BNST stimulation are the result of activation of fibers of passage connecting $\mathrm{CeA}$ to $\mathrm{CeA}$ through the anterior commissure. To investigate the role of the BNST in in vivo calling, focal electrolytic lesions were made in the BNST. The behavioral effects of electrolytic damage to the BNST (Fig. 6B) differed from those observed after damage to the CeA. The most pronounced effect of BNST lesions was a deficit in spontaneous calling. Playback experiments require that a male vocalize spontaneously. Beginning 1 week after surgery, if a male failed to vocalize during the first behavioral trial, then he was tested again the next day and then twice a week for the following 2 weeks until he called spontaneously. Whereas frogs with sham lesions always called during the first trial after surgery and CeA-lesioned frogs required an average 1.3 trials, frogs with BNST lesions required an average of 2.7 trials, significantly longer (Kruskal-Wallis, Dunn's multiple comparisons, $\mathrm{H}=11.50, p=0.003)$. Two of 9 BNST-lesioned frogs failed to call spontaneously even after 6 trials, 3 weeks of testing, and did not respond to multiple attempts to stimulate calling with acoustic playbacks. In BNST-lesioned males, the rate of spontaneous vocalization, measured as the rate of calling in 15 

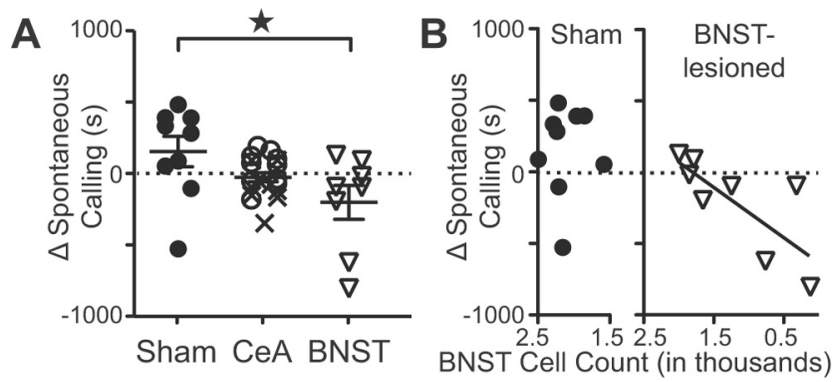

-Sham $\nabla$ BNST ×CeA-IBO oCeA-Electrolytic
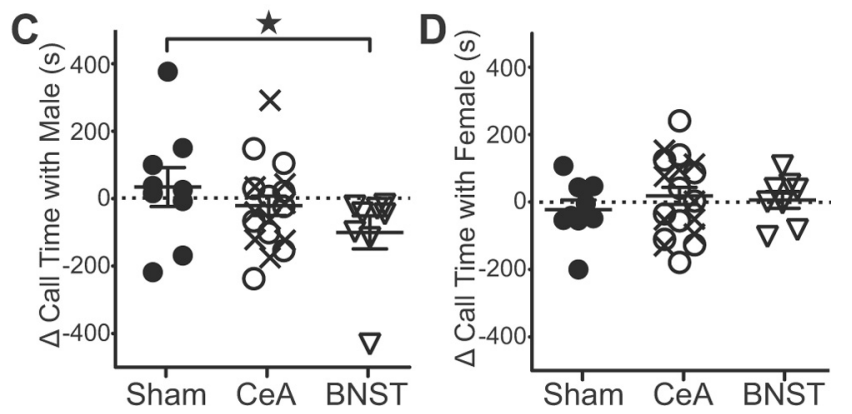

Figure 8. Vocalization rates decrease after BNST lesion. $\boldsymbol{A}$, The prelesion to postlesion change in the time of spontaneous vocalization differed for sham- and BNST-lesioned males. Star and brackets indicate significantly different populations (Kruskal-Wallis $\mathrm{K}=5.756, p=$ 0.053). $\boldsymbol{B}$, The prelesion to postlesion change in the time of spontaneous vocalization was correlated with the number of cells remaining in the BNST (Pearson's $r=0.78, p=0.001$ ). $\boldsymbol{C}$, The prelesion to postlesion change in the time spent vocalizing when paired with a male differed for sham- and BNST-lesioned males. Star and brackets indicate significantly different populations (Kruskal-Wallis $\mathrm{K}=7.683, p=0.0215$ ). $\boldsymbol{D}$, There was no prelesion to postlesion difference in the time spent vocalizing with a female for sham-, CeA-, or BNST-lesioned males.

min without acoustic stimulation, was less than sham-lesioned males (Kruskal-Wallis, Dunn's multiple comparisons, $\mathrm{H}=$ 12.95, $p=0.0047$; Fig. 8A). The prelesion to postlesion decrease in vocalization was greatest after BNST lesion (Kruskal-Wallis, Dunn's multiple comparisons, $\mathrm{H}=8.246, p=0.0412$ ), and this decrease in vocalization rate was correlated with the number of cells remaining in the BNST after lesion (Pearson's $r=0.78, p=$ 0.001; Fig. 8B).

To determine whether BNST-lesioned frogs called less because they were simply unable to vocalize, we paired lesioned frogs with a female or male X. laevis. The failure to vocalize was only observed when the frogs were alone. Even BNST-lesioned frogs that did not vocalize spontaneously or respond to playbacks produced advertisement and answer calls when paired with other frogs. When paired with females, BNST-lesioned males performed advertisement and answer calls at prelesion levels (Wilcoxon, advertisement, $p=0.6406$; answer, $p=0.2930$; time spent calling, $p=0.7402$; Fig. $8 C$ ). When paired with males, however, BNST-lesioned frogs called significantly less than they did before lesion (Wilcoxon, $p=0.008$ ) and less than sham-lesioned males (Kruskal-Wallis, Dunn's multiple comparisons, $\mathrm{H}=9.243, p=$ 0.026; Fig. $8 D$ ). The prelesion to postlesion decrease in calling with males was correlated with the cell count in the BNST (Pearson's $r=0.55, p=0.04)$.

The BNST-lesioned frogs that resumed spontaneous calling $(n=7)$ no longer responded to rapping with increased calling (Freidman $\mathrm{Q}=4.333, p=0.1416)$ or to ticking (Friedman $\mathrm{Q}=$ $0.0, p=1.0$ ) with decreased calling. In contrast to CeA lesions, there was no correlation between BNST cell count and the time to suppression or call resumption after suppression (Pearson's, $p>$ 0.05 ), or overall difference between BNST-lesioned and shamlesioned males in the timing of suppression or resumption of calling in response to any of the presented calls (Friedman, $p>$ 0.05). BNST-lesioned males responded like CeA- and shamlesioned males to playbacks of male advertisement calling with vocal suppression (Friedman $\mathrm{Q}=10.33, p=0.002$ ). However, unlike males electrolytically lesioned in the CeA, BNST-lesioned males did not show suppression to rapping.

\section{Discussion}

Our results reveal that vocal communication in $X$. laevis is influenced by a neural circuit that passes through the CeA, connecting the CT and the ascending auditory pathway to DTAM in the hindbrain VPG (Fig. 3G). Electrical stimulation of the CeA or BNST ex vivo can initiate vocal nerve activity and even fictive calling. In vivo damage to the CeA impairs the male's ability to respond appropriately to playbacks of the female fertility call, rapping, with increased calling and produces responses to female calls that resemble responses to male calls: prolonged suppression. When paired with an actual male or female, damage to the CeA decreases production of the socially appropriate answer call. This evidence suggests that the CeA is involved in the perception and classification of social cues that convey sex and reproductive state. In contrast, the BNST, which does not receive direct auditory input, plays a role in the initiation of vocal behavior. These results clearly demonstrate the importance of the anuran extended amygdala in vocal social interactions.

Although homologous in terms of embryology, chemoarchitecture, and some aspects of connectivity, there are some significant differences in the organization of the anuran extended amygdala compared with that of mammals (Moreno and González, 2005, 2007; Laberge et al., 2006; Moreno et al., 2012). Generally, thalamic sensory inputs are principally directed to subpallial regions in anamniotes (such as anurans), but to pallial derivatives in amniotes (such as mammals and birds) (ten Donkelaar, 1999; Butler, 2008). Following this pattern, in the anuran amygdala the subpallial derivative CeA, rather than the pallial basolateral nucleus (basolateral amygdala) as in mammals, is the principal recipient of sensory input from dorsal thalamic nuclei. Because it is both the principal recipient of sensory input from dorsal thalamic nuclei and the major efferent output center of the amygdaloid complex in frogs, the anuran CeA is likely to be functionally comparable with both basolateral amygdala and CeA in mammals. Compared with the mammalian arrangement where the primary input and output layers of the amygdaloid complex are distinct neural populations that are presumably independently capable of undergoing plasticity, the anuran amygdaloid complex may be limited in terms of its ability to flexibly associate external stimuli and contexts with different behavioral outputs resulting from its single combined input-output layer.

\section{What is the role of the extended amygdala in X. laevis social behavior?}

Across vertebrates, the CeA and BNST are thought to participate in a conserved network that regulates social behaviors (Newman, 1999; Goodson and Kabelik, 2009). Our findings in X. laevis contribute to this consensus. However, the differences between the behavioral effects of damage to the CeA or BNST suggest a specialized role for each nucleus in social vocal behavior. The BNST may influence social behavior through indirect pathways to the VPG, some shared with the CeA (Endepols and Walkowiak, 2001; Goodson and Bass, 2002; Kittelberger et al., 2006). Targets of the 
CeA and BNST that have been generally implicated in the coordination of motor and social behavior include the posterior thalamic nucleus and regions within the midbrain tegmentum (including the substantia nigra, pedunculopontine tegmental nucleus, and lateral dorsal tegmental nucleus) for the CeA, and hypothalamic areas (including the preoptic area and lateral hypothalamus), habenula, and lateral dorsal tegmental nucleus for the BNST (Marín et al., 1998a; Moreno and González, 2005; Moreno et al., 2012).

\section{The role of the CeA in vocal interactions}

Alterations in vocal responses after CeA lesion may reflect an inability to perceive or distinguish behaviorally relevant social signals. The calls of $X$. laevis include acoustic cues that communicate sex and behavioral state (Vignal and Kelley, 2007; Elliott and Kelley, 2007). When pairs of males interact vocally, one is muted and this vocal suppression is persistent (Tobias et al., 1998, 2004, 2010). Males are also vocally suppressed by female ticking, but this suppression is transient and, upon habituation, males resume advertisement calling (Elliott and Kelley, 2007). Males respond to the sexually receptive female call (rapping) with increased calling, but damage to the CeA abolishes this response, and electrolytic lesions result in call suppression during rapping playbacks. CeA-lesioned males suppress calling more rapidly and resume calling later during playbacks of rapping or ticking, often demonstrating persistent suppression of vocalization by these female calls. The CeA-lesioned male responds to the calls of a female as though presented with a vocally dominant male. The $\mathrm{CeA}$ is the principal auditory recipient nucleus within the anuran amygdala. Current evidence suggests that the mammalian amygdala is responsible for processing socially relevant qualities in communication calls (Naumann and Kanwal, 2011; Peterson and Wenstrup, 2012). If CeA damage in X. laevis results in the inability to distinguish male and female vocal signals, then decreased vocal responsiveness and answer call production would be an effect of impaired processing of social cues after CeA lesion.

CeA lesion may also impair the ability to modulate motor responses to social cues. Damage to the CeA results in deficits in answer calling, which could be the result of the loss of forebrain input to the VPG. Nucleus DTAM determines trill length (Zornik et al., 2010), and the ratio of fast to slow trill length is the feature that distinguishes advertisement and answer calling (Tobias et al., 1998). CeA input to DTAM could reconfigure the VPG to generate answer calls by modulating the cellular and synaptic processes responsible for trill duration. Thus, behavioral changes observed after damage to the CeA likely result from changes in the perception of sensory stimuli and/or the initiation of motor responses.

\section{The role of the BNST in vocal initiation}

Damage to the BNST resulted in a decrease in spontaneous calling. BNST-lesioned frogs called in the presence of a female or male frog, but only a female frog elicited prelesion levels of advertisement and answer calling. Two of 9 BNST-lesioned frogs called only in the presence of another frog, suggesting that playback stimuli were not as effective as multimodal stimuli in activating the VPG. These results are consistent with reports of the loss of antiphonal calling to playbacks in Hyla versicolor after lesioning the ventral striatum, which includes both the BNST and CeA (Walkowiak et al., 1999). The BNST projects directly to the dorsal raphe nucleus in X. laevis (Moreno and González, 2012), a nucleus thought to participate in vocal control based on anatomical and pharmacological observations ex vivo (Brahic and Kelley, 2003; Rhodes et al., 2007; Yu and Yamaguchi, 2009, 2010), but not to the VPG directly. Our observations of lesioned males suggest that the BNST is involved in the initiation of vocalization during sexually receptive states, and this may be accomplished through an indirect connection to the VPG through the raphe or hypothalamus. The BNST expresses receptors for gonadotropin and thus may also participate in the central action of this hormone in stimulating advertisement calling (Yang et al., 2007). These findings are consistent with a role for the BNST in the expression of behavioral state-dependent behavior but not with the rapid modulation of the VPG to, for example, produce different types of calls.

\section{Distinct processing pathways for female vocal communication sounds}

When a male $X$. laevis is presented with a change in the acoustic environment, a vocal pause may be a default response, akin to acoustic startle or freezing. Call suppression is an extended vocal pause (Methods 2.8) (Elliott and Kelley, 2007) observed in sham males during advertisement and female ticking playbacks (Fig. $5 A$ ). That vocal suppression per se remains after amygdalar lesions suggests the possibility that auditory information sufficient for vocal pause and call suppression can access the VPG from thalamic or subthalamic nuclei (Lee et al., 1996; Luksch and Walkowiak, 1998; Endepols and Walkowiak, 2001). The CeA, in this case, would facilitate the resumption of calling, rapidly after vocal pause to rapping or slowly after brief suppression to ticking. Because the majority of the CeA cells are GABAergic (Brox et al., 2003), the proposed mechanism of CeA-induced call initiation would be the disinhibition of calling circuitry. Indirect forebrain input to the VPG may also share this mechanism (Endepols and Walkowiak, 1999).

The extended amygdala can potentiate the acoustic startle response during periods of fear or anxiety (Liang et al., 1992; Koch, 1999; Davis and Shi, 2006), and damage to the CeA impairs the ability to evaluate potential threats (Amaral et al., 2003). Our findings suggest a complementary role: to evaluate potential mates using the valent qualities of acoustic communication calls and then generate an appropriate response, in this case by either initiating calling or biasing the VPG toward the production of answer calls. In this view, the extended amygdala is broadly involved in the evaluation of sensory stimuli, and the generation of behavior fitting the environment and internal state of the animal (Belova et al., 2008; Adolphs, 2010; Morrison and Salzman, 2010). The deficits in call initiation or answer call production observed after BNST or CeA lesions in X. laevis support this role and provide a powerful model system to explore the neural circuitry for social recognition.

\section{References}

Adolphs R (2010) What does the amygdala contribute to social cognition? Ann N Y Acad Sci 1191:42-61. CrossRef Medline

Amaral DG, Bauman MD, Capitanio JP, Lavenex P, Mason WA, MauldinJourdain ML, Mendoza SP (2003) The amygdala: is it an essential component of the neural network for social cognition? Neuropsychologia 41:517-522. CrossRef Medline

Belova MA, Paton JJ, Salzman CD (2008) Moment-to-moment tracking of state value in the amygdala. J Neurosci 28:10023-10030. CrossRef Medline

Benaglia T, Chauveau D, Hunter DR, Young DS (2009) mixtools: an R package for analyzing mixture models. J Statistical Software 32:1-29.

Brahic CJ, Kelley DB (2003) Vocal circuitry in Xenopus laevis: telencephalon to laryngeal motor neurons. J Comp Neurol 464:115-130. CrossRef Medline

Brox A, Puelles L, Ferreiro B, Medina L (2003) Expression of the genes GAD67 and Distal-less-4 in the forebrain of Xenopus laevis confirms a 
common pattern in tetrapods. J Comp Neurol 461:370-393. CrossRef Medline

Butler AB (2008) Evolution of the thalamus: a morphological and functional review. Thalamus Related Systems 4:35-58.

Carr CE, Edds-Walton PL (2008) Vertebrate auditory pathways. In: Handbook of the senses, Vol 1, Audition (Hoy R, Dallos P, Oertel D, eds), pp 499-523. Oxford: Elsevier.

Chambers RA, Lipska BK (2011) A method to the madness: producing the neonatal ventral hippocampal lesion rat model of schizophrenia. In: Animal models of schizophrenia and related disorders, neuromethods (O’Donnell P, ed), pp 1-24. New York: Humana, Springer.

Davis M, Shi C (2006) The extended amygdala: are the central nucleus of the amygdala and the bed nucleus of the stria terminalis differentially involved in fear versus anxiety? Ann N Y Acad Sci 877:281-291. CrossRef Medline

Edwards CJ, Kelley DB (2001) Auditory and lateral line inputs to the midbrain of an aquatic anuran: neuroanatomic studies in Xenopus laevis. J Comp Neurol 438:148-162. CrossRef Medline

Elliott TM, Kelley DB (2007) Male discrimination of receptive and unreceptive female calls by temporal features. J Exp Biol 210:2836-2842. CrossRef Medline

Endepols H, Walkowiak W (1999) Influence of descending forebrain projections on processing of acoustic signals and audiomotor integration in the anuran midbrain. Eur J Morphol 37:182-184. CrossRef Medline

Endepols H, Walkowiak W (2001) Integration of ascending and descending inputs in the auditory midbrain of anurans. J Comp Physiol A Neuroethol Sens Neural Behav Physiol 186:1119-1133. CrossRef Medline

Goodson JL, Bass AH (2002) Vocal-acoustic circuitry and descending vocal pathways in teleost fish: convergence with terrestrial vertebrates reveals conserved traits. J Comp Neurol 448:298-322. CrossRef Medline

Goodson JL, Kabelik D (2009) Dynamic limbic networks and social diversity in vertebrates: from neural context to neuromodulatory patterning. Front Neuroendocrinol 30:429-441. CrossRef Medline

Johansen JP, Cain CK, Ostroff LE, LeDoux JE (2011) Molecular mechanisms of fear learning and memory. Cell 147:509-524. CrossRef Medline

Kelley DB (1980) Auditory and vocal nuclei in the frog brain concentrate sex hormones. Science 207:553-555. CrossRef Medline

Kelley DB (1981) Locations of androgen-concentrating cells in the brain of Xenopus laevis: autoradiography with ${ }^{3} \mathrm{H}$-dihydrotestosterone. J Comp Neurol 199:221-231. CrossRef Medline

Kittelberger JM, Land BR, Bass AH (2006) Midbrain periaqueductal gray and vocal patterning in a Teleost fish. J Neurophysiol 96:71-85. CrossRef Medline

Koch M (1999) The neurobiology of startle. Prog Neurobiol 59:107-128. CrossRef Medline

Kohler C, Schwarcz R (1983) Comparison of ibotenate and kainate neurotoxicity in rat brain: a histological study. Neuroscience 8:819-835. CrossRef Medline

Laberge F, Mühlenbrock-Lenter S, Grunwald W, Roth G (2006) Evolution of the amygdala: new insights from studies in amphibians. Brain Behav Evol 67:177-187. CrossRef Medline

Lee Y, López DE, Meloni EG, Davis M (1996) A primary acoustic startle pathway: obligatory role of cochlear root neurons and the nucleus reticularis pontis caudalis. J Neurosci 16:3775-3789. Medline

Liang KC, Melia KR, Campeau S, Falls WA, Miserendino MJ, Davis M (1992) Lesions of the central nucleus of the amygdala, but not the paraventricular nucleus of the hypothalamus, block the excitatory effects of corticotropin-releasing factor on the acoustic startle reflex. J Neurosci 12:2313-2320. Medline

Luksch H, Walkowiak W (1998) Morphology and axonal projection patterns of auditory neurons in the midbrain of the painted frog, Discoglossus pictus. Hear Res 122:1-17. CrossRef Medline

Marín O, Smeets WJ, González A (1998a) Basal ganglia organization in amphibians: evidence for a common pattern in tetrapods. Prog Neurobiol 55:363-397. CrossRef Medline

Marín O, Smeets WJ, González A (1998b) Basal ganglia organization in amphibians: chemoarchitecture. J Comp Neurol 392:285-312. CrossRef Medline

Moreno N, González A (2005) Central amygdala in anuran amphibians: neurochemical organization and connectivity. J Comp Neurol 489: 69-91. CrossRef Medline

Moreno N, González A (2007) Evolution of the amygdaloid complex in vertebrates, with special reference to the anamnio-amniotic transition. J Anat 211:151-163. CrossRef Medline

Moreno N, Morona R, López JM, Domínguez L, Joven A, Bandín S, González A (2012) Characterization of the bed nucleus of the stria terminalis in the forebrain of anuran amphibians. J Comp Neurol 520:330-363. CrossRef Medline

Morrison SE, Salzman CD (2010) Re-valuing the amygdala. Curr Opin Neurobiol 20:221-230. CrossRef Medline

Naumann RT, Kanwal JS (2011) Basolateral amygdala responds robustly to social calls: spiking characteristics of single unit activity. J Neurophysiol 105:2389-2404. CrossRef Medline

Newman SW (1999) The medial extended amygdala in male reproductive behavior: a node in the mammalian social behavior network. Ann N Y Acad Sci 877:242-257. CrossRef Medline

O'Connell LA, Hofmann HA (2011) The vertebrate mesolimbic reward system and social behavior network: a comparative synthesis. J Comp Neurol 519:3599-3639. CrossRef Medline

Peterson DC, Wenstrup JJ (2012) Selectivity and persistent firing responses to social vocalizations in the basolateral amygdala. Neuroscience 217:154-171. CrossRef Medline

Rhodes HJ, Yu HJ, Yamaguchi A (2007) Xenopus vocalizations are controlled by a sexually differentiated hindbrain central pattern generator. J Neurosci 27:1485-1497. CrossRef Medline

Rodrigues SM, LeDoux JE, Sapolsky RM (2009) The influence of stress hormones on fear circuitry. Annu Rev Neurosci 32:289-313. CrossRef Medline

Schwarcz R, Hökfelt T, Fuxe K, Jonsson G, Goldstein M, Terenius L (1979) Ibotenic acid-induced neuronal degeneration: a morphological and neurochemical study. Exp Brain Res 37:199-216. Medline

Spiwoks-Becker I, Vollrath L, Seeliger MW, Jaissle G, Eshkind LG, Leube RE (2001) Synaptic vesicle alterations in rod photoreceptors of synaptophysindeficient mice. Neuroscience 107:127-142. CrossRef Medline

ten Donkelaar HJ (1999) Some introductory notes on the organization of the forebrain in tetrapods. Eur J Morphol 37:73-80. CrossRef Medline

Tobias ML, Viswanathan SS, Kelley DB (1998) Rapping, a female receptive call, initiates male-female duets in the South African clawed frog. Proc Natl Acad Sci U S A 95:1870-1875. CrossRef Medline

Tobias ML, Barnard C, O’Hagan R, Horng SH, Rand M, Kelley DB (2004) Vocal communication between male Xenopus laevis. Anim Behav 67:353365. CrossRef Medline

Tobias ML, Corke A, Korsh J, Yin D, Kelley DB (2010) Vocal competition in male Xenopus laevis frogs. Behav Ecol Sociobiol 64:1791-1803. CrossRef Medline

Valtorta F, Pennuto M, Bonanomi D, Benfenati F (2004) Synaptophysin: leading actor or walk-on role in synaptic vesicle exocytosis? Bioessays 26:445-453. CrossRef Medline

Vignal C, Kelley D (2007) Significance of temporal and spectral acoustic cues for sexual recognition in Xenopus laevis. Proc Biol Sci 274:479-488. CrossRef Medline

Walkowiak W, Berlinger M, Schul J, Gerhardt HC (1999) Significance of forebrain structures in acoustically guided behavior in anurans. Eur J Morphol 37:177-181. CrossRef Medline

Wetzel DM, Kelley DB (1983) Androgen and gonadotropin effects on male mate calls in South African clawed frogs, Xenopus laevis. Horm Behav 17:388-404. CrossRef Medline

Yamaguchi A, Kelley DB (2000) Generating sexually differentiated vocal patterns: laryngeal nerve and EMG recordings from vocalizing male and female african clawed frogs (Xenopus laevis). J Neurosci 20:1559-1567. Medline

Yang EJ, Nasipak BT, Kelley DB (2007) Direct action of gonadotropin in brain integrates behavioral and reproductive functions. Proc Natl Acad Sci U S A 104:2477-2482. CrossRef Medline

Yu HJ, Yamaguchi A (2009) 5-HT2C-like receptors in the brain of Xenopus laevis initiate sex-typical fictive vocalizations. J Neurophysiol 102:752-765. CrossRef Medline

Yu HJ, Yamaguchi A (2010) Endogenous serotonin acts on 5-HT2C-like receptors in key vocal areas of the brain stem to initiate vocalizations in Xenopus laevis. J Neurophysiol 103:648-658. CrossRef Medline

Zornik E, Kelley DB (2007) Breathing and calling: neuronal networks in the Xenopus laevis hindbrain. J Comp Neurol 501:303-315. CrossRef Medline Zornik E, Kelley DB (2008) Regulation of respiratory and vocal motor pools 
in the isolated brain of Xenopus laevis. J Neurosci 28:612-621. CrossRef Medline

Zornik E, Kelley DB (2011) A neuroendocrine basis for the hierarchical control of frog courtship vocalizations. Front Neuroendocrinol 32:353-366. CrossRef Medline
Zornik E, Katzen AW, Rhodes HJ, Yamaguchi A (2010) NMDARdependent control of call duration in Xenopus laevis. J Neurophysiol 103: 3501-3515. CrossRef Medline

Zornik E, Yamaguchi A (2012) Coding rate and duration of vocalizations of the frog, Xenopus laevis. J Neurosci 32:12102-12114. CrossRef Medline 